\title{
3D Hermite Transform Optical Flow Estimation in Left Ventricle CT Sequences
}

\author{
Carlos Mira ${ }^{1, *(\mathbb{D})}$, Ernesto Moya-Albor ${ }^{2}(\mathbb{D})$, Boris Escalante-Ramírez ${ }^{1, *([)}$, Jimena Olveres ${ }^{1}$ (1) , \\ Jorge Brieva $^{2}$ (I) and Enrique Vallejo ${ }^{3}$ \\ 1 Facultad de Ingeniería, Universidad Nacional Autónoma de México, Ciudad de México 04510, Mexico; \\ jolveres@gmail.com \\ 2 Facultad de Ingeniería, Universidad Panamericana, Augusto Rodin 498, Ciudad de Mexico 03920, Mexico; \\ emoya@up.edu.mx (E.M.-A.); jbrieva@up.edu.mx (J.B.) \\ 3 Centro Médico ABC, Ciudad de México 01120, Mexico; vallejo.enrique@gmail.com \\ * Correspondence: miragnlz@live.com (C.M.); boris@unam.mx (B.E.-R.)
}

Received: 22 November 2019; Accepted: 10 January 2020; Published: 21 January 2020

check for updates

\begin{abstract}
Heart diseases are the most important causes of death in the world and over the years, the study of cardiac movement has been carried out mainly in two dimensions, however, it is important to consider that the deformations due to the movement of the heart occur in a three-dimensional space. The $3 D+t$ analysis allows to describe most of the motions of the heart, for example, the twisting motion that takes place on every beat cycle that allows us identifying abnormalities of the heart walls. Therefore, it is necessary to develop algorithms that help specialists understand the cardiac movement. In this work, we developed a new approach to determine the cardiac movement in three dimensions using a differential optical flow approach in which we use the steered Hermite transform (SHT) which allows us to decompose cardiac volumes taking advantage of it as a model of the human vision system (HVS). Our proposal was tested in complete cardiac computed tomography $(\mathrm{CT})$ volumes $(3 D+t)$, as well as its respective left ventricular segmentation. The robustness to noise was tested with good results. The evaluation of the results was carried out through errors in forwarding reconstruction, from the volume at time $t$ to time $t+1$ using the optical flow obtained (interpolation errors). The parameters were tuned extensively. In the case of the 2D algorithm, the interpolation errors and normalized interpolation errors are very close and below the values reported in ground truth flows. In the case of the $3 \mathrm{D}$ algorithm, the results were compared with another similar method in 3D and the interpolation errors remained below 0.1. These results of interpolation errors for complete cardiac volumes and the left ventricle are shown graphically for clarity. Finally, a series of graphs are observed where the characteristic of contraction and dilation of the left ventricle is evident through the representation of the 3D optical flow.
\end{abstract}

Keywords: bio-inspired computing; motion estimation; optical flow; differential method; steered hermite transform; cardiac CT imaging; algorithms

\section{Introduction}

Cardiovascular diseases (CVDs) take the lives of 17.9 million people every year, $31 \%$ of all global deaths, this represents the number one cause of death globally, more people die annually from CVDs than from any other cause [1]. Heart diseases as myocardial infarction, ischemia or hypertrophy can be characterized by analyzing the dynamics of the heart. During the cardiac cycle (contraction "systole" and relaxation "diastole" of the heart), the motion wall estimation can be used to recognize those pathologies. The acquisition of cardiac volumes has allowed quantifying relevant left ventricular (LV) parameters such as its volume, strain, twist, and desynchrony [2]. Nowadays, there are 
diagnostic imaging techniques to characterize cardiac anatomy and function, as such echocardiography, cardiac Magnetic Resonance Imaging (MRI), cardiac Computed Tomography (CT), cardiac Positron Emission Tomography (PET), and coronary angiography [3]. Where the cardiac CT technique has certain advantages with respect other: a higher resolution that ultrasound, it is more accessible than MRI and it is a non-invasive a fast imaging option [4]. Cardiac CT allows acquiring three-dimensional morphological images, with motion artifacts minimizes, and showing the heart chambers and the coronary arteries at different planes [5]. It is then possible to acquire good quality cardiac CT data of the heartbeats. Images are acquired overall cardiac cycles to produce the final volume image. Due to the complexity of the heart motion, it is still hard for the physician to estimate the 3D motion during the exam, thus, it is necessary to develop computational analysis tools to aid in the diagnosis process.

The human heart is a complex organ in terms of anatomy and physiology, the estimation of its movement is an important task to understand its mechanism and to assist in the medical diagnosis. Different image processing techniques can be applied to calculate and to observe the motion of the heart, for example, the optical flow estimation is a method used in those situations where the correspondence between the pixels, within an image sequence, is required. The optical flow methods compute an approximation to the 2D motion in an image sequence from spatiotemporal patterns of image intensity [6]. Over the years, state-of-the-art algorithms for optical flow can be summarized in Nagel [7] who identified the common rigorous restrictions [8-10], as well as smoothing restrictions for the optical flow solution $[7,11,12]$. On the other hand, Barron et al. [6], categorized the optical flow in four groups: differential techniques [7-9,11,13,14], region-based matching [15,16], energy-based methods [17,18] and phase-based techniques [19]. Sun et al. [20] suggested that there have been few changes in the typical formulation given by Horn and Schunck [11]. Most of research work on optical flow has been carried out in $2 D+t$. Many of them claim to be $3 \mathrm{D}$, but they really are $2 D+t$. Some of them, use 2D projections to obtain a 3D representation, in applications such as tracking traffic [21], in methods used for quantitative motion estimation of biological structures in light microscope [22], estimation of 3D geometry and 3D motion using spatiotemporal gradients [23] or emotion recognition from 3D videos [24]. 2D optical flow estimation has been used in the heart analysis to identify patients with some diseases, recent works using 2D optical flow cover topics such as motion estimation in cardiac fluorescence imaging [25], and as the automatic localization of the heart from cine MRI [26]. Some optical flow methods have used image models inspired by nature, for example Gabor filters $[18,19,27-30]$. The optical flow is fundamentally different than tracking because a complete set of correspondences between the pixel levels in an image (or volume) is obtained. The optical flow is used to calculate dense trajectories, provides more freedom and information about the data in which the movement is being estimated, a priori models are not needed and even more, it can also be used to develop deformable based-model tracking algorithms.

The Hermite transform (HT) has been an image model used to describe the local constraints of the Horn and Schunck approach. Liu et al. [27] derived a six-parameter non-affine optical flow model, which is solved with high-order Hermite polynomial filtered data. In [28], Silvan et al. showed that through a linear mapping of 3D Hermite coefficients by specific projection functions, we could obtain the Hermite transform coefficients of local projections. Furthermore, Moya et al. [29] used the steered Hermite coefficients like local motion restrictions, found in current methods, to define a differential estimation method.

One disadvantage of the 2D cardiac movement analysis is that it is constrained by geometry-dependent reference directions of deformation (i.e., radial, circumferential, and longitudinal). In this sense, a 3D cardiac movement analysis may overcome such limitations by referencing the intrinsic directions of deformation [31]. Thus, to identify altered ventricular function in patients with CVD, a 3D left ventricular (LV) deformation analysis is more suitable since it represents contributions from counter-directional, helically arranged fibers shortening and thickening throughout the cardiac cycle [32]. Research on the measurement of cardiac motion has been commonly made in $2 D+t$ [33] but this analysis should be done in $3 D+t$ to enable us to describe the true motions of the heart, for example, 
the twisting motion that takes place on every beat cycle. Compared to the 2D analysis, the 3D analysis has not received much attention, although there are currently working groups analyzing optical flow in 3D mainly using ultrasound images.

About the estimation of 3D optical flow in general, some works have similarities with at least one of the aspects of this article, thereby, in [34] they use a 3D model of the human body and motion captured data to synthesize flow fields and train a convolutional neural network (CNN) to estimate human flow fields from pairs of images. In [35] a steerable filter-based algorithm is formulated, in its simplest form, for estimating 3D flow in sequences of volumetric or point-cloud data.

In [36] they present an approach for real-time respiratory motion estimation in image-guided interventions by employing contrast-invariant feature descriptors. Yoon et al. [37] presented a method for motion estimation applied to cone-beam CT, their work uses an energy functional, which includes as terms: a data fidelity, a regularization term, and the optical flow restriction. On the other hand, Jungwon et al. [38] used the optical flow estimation to calculate the local motion, allowing a 3D segmentation extension. Their model includes a shape distortion over time term, allowing segmenting and tracking the lung nodules. In [39], an implementation based on the optical flow algorithm from Farnebäck (2003) is used to create 3D freehand ultrasound but with reconstructions from 2D without external tracking, using deep learning.

Several methods have been used to estimate the optical flow of the endocardial wall motion [40]. In [41], a global anatomically constrained affine optical flow tracking was used to track the end-diastole left ventricle surface throughout the cardiac cycle. For [42], this approach first performs 3D segmentation at the end-diastolic frame and then performs tracking over the cardiac cycle using both global (optical flow) and local (block matching) methods. In [43] they claim to have a method for detecting cardiac flow in echocardiography where the sampling planes representing the mitral inflow tract and the left ventricle outflow tract are traced by fusing information from multiple cues, including optical flow, boundary detection, and motion prior. Duan et al. [44], evaluate a correlation-based optical flow algorithm for tracking endocardial surfaces on three-dimensional ultrasound data, also in [45] they built a truly 3D mathematical phantom of cardiac tissue and blood in order to validate the optical flow for quantification of myocardial deformations. Leung et al. [46] track left ventricular borders in 3D echocardiographic sequences by combining differential optical flow with statistical modeling. Zhiang et al. [47] developed an optical flow algorithm based on Thirion's diffusing model [48], also known as the "demons" algorithm and also described an atlas-based geometry pipeline for constructing three-dimensional cubic Hermite finite element meshes of the human heart.

In Table 1 we summarized some of the most recent optical flow motion methods used to extract the motion estimation, either using a $2 D+t$ or $3 D+t$ model, and the differences with the proposed method. In some cases, a 2D optical flow is initially estimated to map it onto a 3D optical flow.

The present article is an extension of our previous work published in [49]. In that work, we proposed the three-dimensional optical flow estimation using the 3D steered Hermite transform and we compared our approach with the 3D Horn-Schunck method. In contrast, the current work is compared with a multiresolution Horn and Schunck approach reported by Sun et al. [20], moreover, in this work, we perform a depth analysis about the optimal parameters of the method proposed and we focused the Section 5.2.1 to analyzed the 3D optical flow estimation of the left ventricle, first, showing its 3D segmentation and showing the advantages of our approach compared with the 3D version of the method of Sun et al. [20], and then, showing the 3D motion of the left ventricle in different cardiac cycle and a whole cardiac cycle, highlighting the corresponding contraction and relaxation movements present in each phase of the cardiac cycle. It should be mentioned that similar $2 \mathrm{D}$ algorithms using the Hermite transform have already been presented in $[29,33]$ but with the main disadvantage of consuming a lot of computing time. 
Table 1. $2 D / 3 D+t$ optical flow estimation approaches.

\begin{tabular}{ccccc}
\hline Paper & $\begin{array}{c}\text { OF Model } \\
(2 D / 3 D+t)\end{array}$ & Method & Application & Evaluation Metric \\
\hline Proposed method & 3D & $\begin{array}{c}\text { Using the 3D Steered } \\
\text { Hermite Transform }\end{array}$ & $\begin{array}{c}\text { Left ventricle } \\
\text { CT sequences }\end{array}$ & $\begin{array}{c}\text { Interpolation } \\
\text { errors in 3D }\end{array}$ \\
\hline Ranjan et al. [34] & 3D & $\begin{array}{c}\text { A 3D model human } \\
\text { body and a CNN }\end{array}$ & $\begin{array}{c}\text { Estimate human } \\
\text { flow fields }\end{array}$ & End point error \\
\hline Alexiadis et al. [35] & 2D & $\begin{array}{c}\text { Minimizing } \\
\text { a cost functional }\end{array}$ & $\begin{array}{c}\text { 3D flow } \\
\text { estimation }\end{array}$ & $\begin{array}{c}\text { Mean angular error } \\
\text { on synthetic images }\end{array}$ \\
\hline Queiros et al. [41] & 3D & $\begin{array}{c}\text { Anatomically affine } \\
\text { optical flow }\end{array}$ & $\begin{array}{c}\text { Left ventricle } \\
\text { tracking }\end{array}$ & $\begin{array}{c}\text { Distance and } \\
\text { Dice metrics }\end{array}$ \\
\hline Patil et al. [24] & 2D & Farnebäck & $\begin{array}{c}\text { Emotion } \\
\text { recognition }\end{array}$ & $\begin{array}{c}\text { Accuracy } \\
\text { of 6 emotions }\end{array}$ \\
\hline Saleh et al. [26] & 2D & Lucas-Kanade & $\begin{array}{c}\text { Heart } \\
\text { Localization }\end{array}$ & $\begin{array}{c}\text { Accuracy } \\
\text { on localizing }\end{array}$ \\
\hline Baghaie et al. [30] & 2D & Gabor, Schmid and \\
steerable filters & $\begin{array}{c}\text { 2D flow } \\
\text { estimation }\end{array}$ & $\begin{array}{c}\text { Angular and } \\
\text { interpolation } \\
\text { errors }\end{array}$ \\
\hline Rodriguez et al. [25] & 2D & Horn \& Schunck & $\begin{array}{c}\text { Cardiac motion } \\
\text { estimation }\end{array}$ & $\begin{array}{c}\text { Mean square } \\
\text { error }\end{array}$ \\
\hline
\end{tabular}

Revising the algorithm from $2 \mathrm{D}$ to $3 \mathrm{D}$ is not a trivial problem, on top of the additional and necessary computational complexity, and the importance of describing the 3D cardiac movement. We have to describe (based on [50]) and calculate a second local orientation angle from the 3D cartesian coefficients of Hermite to obtain the 3D steered coefficients of Hermite (SHT3D). The data used in this work require a sensitivity analysis of its parameters and a way to validate the results because there aren't annotated volumes. Robust noise tests and calculation of interpolation errors of the volumes used had been carried out. Left ventricular analysis has been of great importance for this article. In this way, the results of the optical flow and the segmentation of such cardiac structure were evaluated.

Figure 1 shows an overview of the proposed method according to the procedures explained in the next sections.

Our approach uses a three-dimensional $(3 D+t)$, that is, it uses the data of the cardiac volumes in a three-dimensional space $(x, y, z)$, which change over time during the entire cardiac cycle; a modified version of Sun et al. [20], which, in contradistinction of Horn and Schunck's approach [11], uses an incremental multiresolution technique to estimate large displacements, where the optical flow at a coarse level is extrapolated to warp the second image at a finer level, combined with the optical flow based on the Hermite transform proposed by Moya et al. [29], that uses the several constraints found in the more accurate optical flow methods. The rest of the paper is organized as follows: Section 2 describes the 3D Hermite transform, Section 3 develops the proposal to obtain 3D optical flow, Section 5 presents the experimental results and discussion of this work, Section 6 is about the results obtained, and finally, Section 7 concludes the paper and presents future work. 


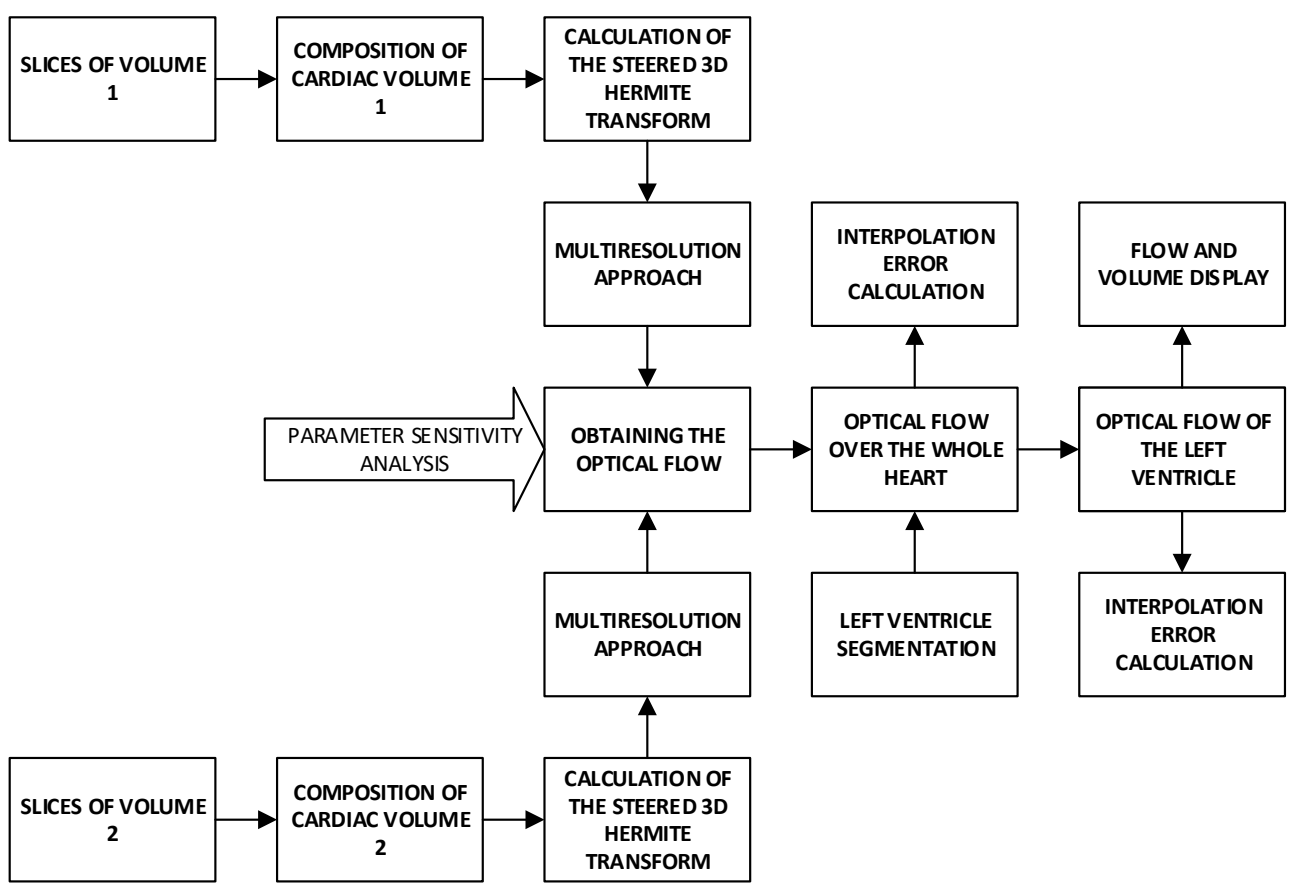

Figure 1. Overview of the proposed method.

\section{The 3D Hermite Transform}

The Hermite transform is a bio-inspired image model, it simulates some of the more relevant properties of the early vision of the human vision system (HVS): the local processing [51] and the Gaussian derivative model of the receptive fields $[50,52,53]$. The SHT provides a very efficient representation of oriented patterns which enables an adaptation to local orientation content at each window position over the image, indicating the direction of the two-dimensional pattern. The Hermite transform uses functions that are derivatives of Gaussians, which have wide applications in the field of computer vision and are a bio-inspired model of the human vision system. In this work, the Hermite transform serves as a theoretical framework to carry out the estimation of cardiac movement in our approach.

Gaussian windows in two dimensions have the property of being rotationally symmetric and spatially separable. Gaussian windows separated by twice the standard deviation, are a good model found for the receptive fields of perception found in psychological experiments [51]. According to the psychophysical model of HVS [52,54], through Gaussian windows, we can decompose an image into several orthogonal polynomials.

An interesting special case of 2D polynomial transforms arises when we have a window function which is separable i.e., $v(x, y)=v(x) v(y)$

For a perceptual standpoint and according to the scale-space theory, we will use a Gaussian window (Figure 2)

$$
v(x, y)=\frac{1}{\sigma \sqrt{\pi}} \exp \left(-\frac{\left(x^{2}+y^{2}\right)}{2 \sigma^{2}}\right)
$$

The direct Hermite transform in 3D (HT3D), is a particular case of the proposal of Martens [50,53], where a signal is localized by an analysis window and this information is expanded using polynomials orthogonal to the window. Polynomials that are orthogonal with respect to the Gaussian window function are defined by [55], so we would use the window:

$$
v(x, y, z)=\frac{1}{\sqrt{\sigma \sqrt{\pi}}} \exp \left(-\frac{\left(x^{2}+y^{2}+z^{2}\right)}{2 \sigma^{2}}\right)
$$




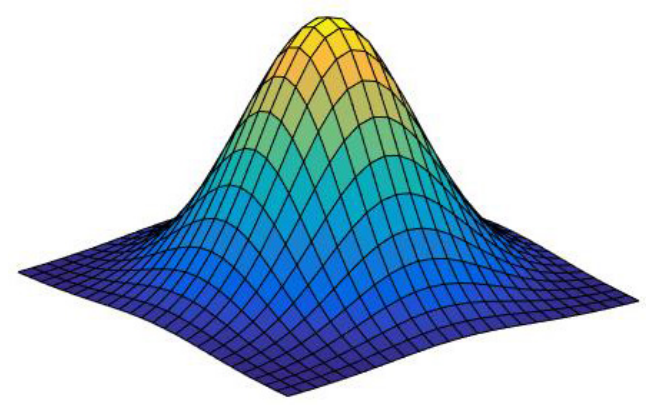

Figure 2. Gaussian window $v(x, y)$.

Physiological experiments consider using overlapping Gaussian windows separated by twice the standard deviation $\sigma$ which are isotropic and that's why we can establish that $\sigma=\sigma_{x}=\sigma_{y}=\sigma_{z}$ in accordance with the overlapping receptive fields of the human visual system [51].

The Hermite cartesian coefficients, $L_{l, m-l, n-m}$, are obtained by convolution of the original signal $L(x, y, z)$ with the analysis filters $D_{l, m-l, n-m}(x, y, z)$ followed by subsampling on a three-dimensional mesh $S$ using Equation (3):

$$
L_{l, m-l, n-m}\left(x_{0}, y_{0}, z_{0}\right)=L(x, y, z) \otimes D_{l, m-l, n-m}(x, y, z),
$$

where $l,(m-l)$ and $(n-m)$ denote the analysis order in $x, y$ and $z$ directions, respectively; $l=0,1, \ldots, m ; m=0,1, \ldots, n ; n=0,1, \ldots, N ; N$ is the maximum order of the expansion that is related to the size of a cubic window of $\mathcal{M} \times \mathcal{M} \times \mathcal{M}$, where $N \leq 2 *(\mathcal{M}-1)$. For large values of $\mathcal{M}$ the discrete cubic kernel reduces to the 3D Gaussian window.

The three-dimensional Hermite filters can be represented by:

$$
D_{l, m-l, n-m}(x, y, z)=G_{l . m-l, n-m}(-x,-y,-z) v^{2}(-x,-y,-z)
$$

wich are separable because the Gaussian window is rotationally symmetric

$$
D_{l, m-l, n-m}(x, y, z)=D_{l}(x) D_{m-l}(y) D_{n-m}(z)
$$

and those can be computed by:

$$
D_{l}(x)=\frac{(-1)^{l}}{\sqrt{2^{l} l !}} \frac{1}{\sigma \sqrt{\pi}} H_{l}\left(\frac{x}{\sigma}\right) \exp \left(-\frac{x^{2}}{\sigma^{2}}\right)
$$

$G_{l, m-l, n-m}(x, y, z)$ are a family of polynomials defined as:

$$
G_{l, m-l, n-m}(x, y, z)=\frac{1}{\sqrt{2^{n} l !(m-l) !(n-m) !}} H_{l}\left(\frac{x}{\sigma}\right) H_{m-l}\left(\frac{y}{\sigma}\right) H_{n-m}\left(\frac{z}{\sigma}\right)
$$

where $H_{l}$ represents the generalized Hermite polynomials given by Rodrigues' formula [56]

$$
H_{l}(x)=(-1)^{l} \exp \left(x^{2}\right) \frac{d^{l}}{d x^{l}} \exp \left(-x^{2}\right)
$$

The recovery process of the original image (inverse Hermite transform in 3D - IHT3D) consists of interpolating the Hermite coefficients through the proper synthesis filters:

$$
\hat{L}(x, y, z)=\sum_{n=0}^{N} \sum_{m=0}^{n} \sum_{l=0}^{m} \sum_{\left(x_{0}, y_{0}, z_{0}\right) \in S} L_{l, m-l, n-m}\left(x_{0}, y_{0}, z_{0}\right) P_{l, m-l, n-m}\left(x-x_{0}, y-y_{0}, z-z_{0}\right)
$$


where $P_{l, m-l, n-m}(x, y, z)$ can be determined by:

$$
P_{l, m-l, n-m}(x, y, z)=\frac{G_{l, m-l, n-m}(x, y, z) v^{2}(x, y, z)}{\sum_{\left(x_{0}, y_{0}, z_{0}\right) \in S} v^{2}\left(x-x_{0}, y-y_{0}, z-z_{0}\right)}
$$

for $l=0, \ldots, m ; m=0, \ldots, n$ and $n=0, \ldots, N$.

From Equation (9), instead of to recover the original volume we obtain an approximation of the original signal $\hat{L}(x, y, z)$, where the quality of this reconstruction improves by increasing the maximum order of the expansion $N$, i.e., the size of the cubic window $\mathcal{M}$ [50]. In terms of the artifacts in the approximated volume $\hat{L}(x, y, z)$, small values of the cubic windows causes "speckles", while high values result in Gibbs-phenomenon-like artifacts such as ringing and blur [57].

Thus, to determined the maximum order or the expansion $N$ and in consequence the size of the cubic window $\mathcal{M}$, in [57] van Dijk and Martens determined that using an expansion of the Hermite transform equal to 3, the reconstructed 2D image will contain the most quantity of AC energy $(84 \%)$ according to Parseval's theorem. In general, with $N \geq 3$ we can obtain a good reconstruction and with much greater values we will obtain a perfect reconstruction of the image.

\section{D Steered Hermite Transform}

The Steered Hermite transform (SHT) is a variant of the HT that adapts to the local orientation of the image [57], it uses rotated filters which are represented as a linear combination of basis filters [58]. The orientation property of these steered Hermite filters is due to the symmetric-radial form of the Gaussian window, thus they can saw as the response of directional derivatives of the Gaussian function.

On the other hand, the SHT describes local 1D patterns in images into a smaller number of coefficients that represent the profile of the pattern perpendicular to its orientation [57].

By projecting the 3D Cartesian Hermite coefficients towards the local orientation angles $\theta$ and $\phi$ (Figure 3), we obtain the Steered Hermite transform in 3D (SHT3D) as shown in Equation (11):

$$
l_{l, m-l, n-m, \theta, \phi}\left(x_{0}, y_{0}, z_{0}\right)=\sum_{m=0}^{n} \sum_{l=0}^{m}\left(L_{l, m-l, n-m}\left(x_{0}, y_{0}, z_{0}\right) \cdot g_{l, m-l}(\theta) \cdot g_{m, n-m}(\phi)\right)
$$

where $l_{l, m-l, n-m, \theta, \phi}\left(x_{0}, y_{0}, z_{0}\right)$ are the 3D steered Hermite coefficients. And

$$
g_{j, k-j}(\varphi)=\sqrt{\left(\begin{array}{c}
k \\
j
\end{array}\right)}\left(\cos ^{j}(\varphi) \sin ^{k-j}(\varphi)\right)
$$

is the cartesian angular function that expresses the directional selectivity of the filter.

To calculate the direction of maximum energy we used the coefficients from Equation (3) and the phase of the gradient given by Equations (13) and (14):

$$
\begin{gathered}
\theta=\arctan \left(\frac{L_{010}}{L_{100}}\right) \\
\phi=\arctan \left(\frac{\sqrt{\left(L_{100}\right)^{2}+\left(L_{010}\right)^{2}}}{L_{001}}\right)
\end{gathered}
$$

where $\left[L_{1,0,0}, L_{0,1,0}, L_{0,0,1}\right](x, y, z)^{\top}$ are a good approximation of the 3D gradient through the Cartesian Hermite coefficients. 


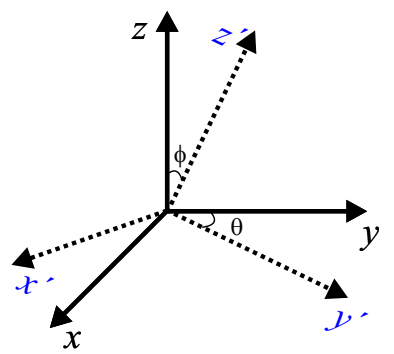

Figure 3. Cartesian coordinates (continuous line), steered coordinates (dotted line) and the angles $\theta$ and $\phi$.

In order to graphically represent the indexes of the 3D Cartesian Hermite coefficients, Figure 4 shows the distribution of order two $(N=2)$ in each direction, in this case 27 coefficients are obtained for each voxel of a volume.

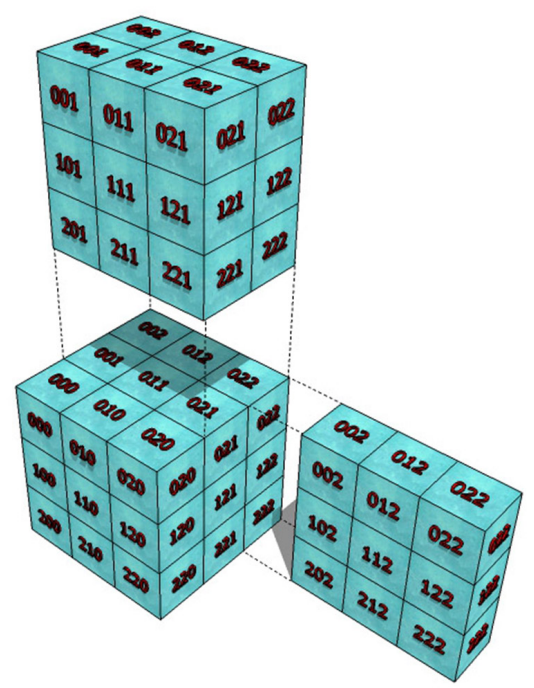

Figure 4. Distribution of the index of Cartesian Hermite coefficients of a second-order voxel.

Figure 5 shows an example of some 3D Steered Hermite coefficients for the left ventricle of a cardiac CT volume, according to Equation (11), where we can see the steered coefficients $l_{000}, l_{100}, l_{010}$ and $l_{001}$.

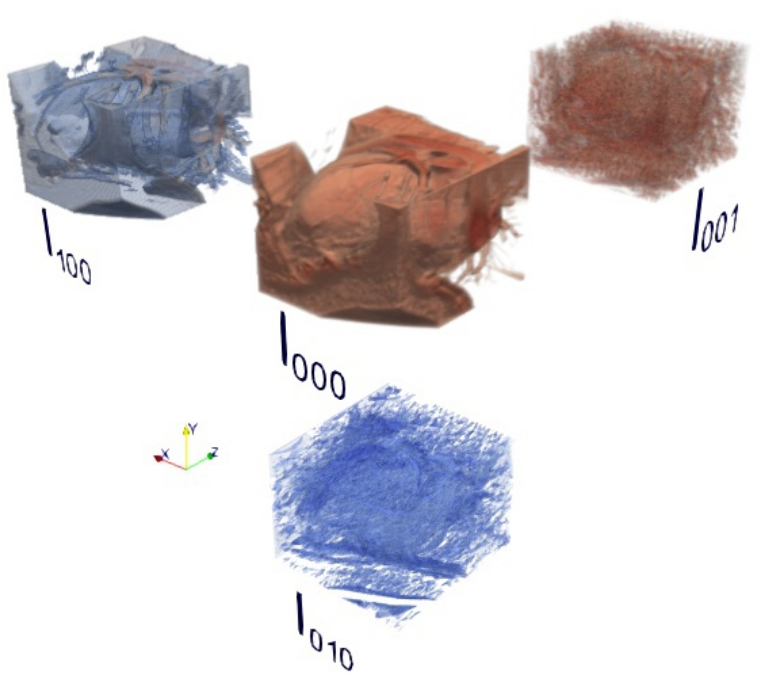

Figure 5. Ensemble of some Steered Hermite coefficients of a cardiac CT volume. 


\section{Optical Flow using the Hermite Transform}

One of the main disadvantages of the classic method of Horn and Schunck [11] is its low accuracy, and because of this, we use a modified version of the method proposed by Sun et al. [20], that solves such obstacle using a multiresolution approach to estimate large displacements. In addition, this modified version is combined using the Hermite Transform with the advantage that it is based on a visual biological model of the images. Consequently, the local constraints of Horn and Schunck are defined using the zero order Hermite coefficient, and the Steered Hermite coefficients are used as high order local descriptors of the visual characteristics of the volumes.

Model

Our approach is based on the multiresolution Horn and Schunck approach reported by Sun et al. [20], it uses the SHT3D to expand the constant intensity constraint and adds the Steered Hermite coefficients constraint as shown in Equation (15):

$$
\left[L_{0}(\mathbf{x}+\mathbf{w})-L_{0}(\mathbf{x})\right]+\gamma\left[\sum_{n=1}^{N} l_{n, \theta, \phi}(\mathbf{x}+\mathbf{w})-\sum_{n=1}^{N} l_{n, \theta, \phi}(\mathbf{x})\right]=0
$$

where $L(\mathbf{x})$ is a volume sequence, with $\mathbf{x}=(x, y, z, t)^{T}$ representing the voxel location within a domain $V ; \mathbf{w}:=(u, v, w, 1)^{T}$ is a vector that defines the displacement $u, v$ and $w$ of each voxel at position $(x, y, z)$ from time $t$ to time $(t+1)$ in the directions $x, y$ and $z$ respectively; and $\gamma$ is a weight parameter that controls the contribution of the high order descriptors. Using the HT optical flow restriction of Equation (15) we defined an energy functional that includes a smooth term to overcome the aperture problem [11] as follows:

$$
\begin{aligned}
\mathrm{E}= & \int_{V}\left(\left[L_{0}(\mathbf{x})_{0}-L_{0}(\mathbf{x}+\mathbf{w}+d \mathbf{w})_{1}\right]^{2}+\right. \\
& \gamma\left[\sum_{n=1}^{N}\left\{l_{n, \theta, \phi}(\mathbf{x})_{0}-l_{n, \theta, \phi}(\mathbf{x}+\mathbf{w}+d \mathbf{w})_{1}\right\}\right]^{2}+ \\
& \left.\alpha|\nabla(\mathbf{w}+d \mathbf{w})|^{2}\right) d \mathbf{x}
\end{aligned}
$$

where $\alpha$ is the weight value of the smoothness term that can get information from neighbors in regions where the intensity gradient is zero (uniform regions of flow).

To simplify the notation $L_{000}(\mathbf{x})=L_{0}(\mathbf{x}), L_{*}(\mathbf{x})_{*}$ is the Cartesian Hermite coefficient $*$ at time $t$ and $L_{*}(\mathbf{x})_{1}$ is the Cartesian Hermite coefficient $*$ at time $t+1$.

Considering linear displacements, the constant intensity term of Equation (15) can be expanded by a Taylor series as shown:

$$
\begin{aligned}
L_{0}(\mathbf{x})_{0}-L_{0}(\mathbf{x}+\mathbf{w}+d \mathbf{w})_{1} \approx L_{0}(\mathbf{x})_{0}-L_{0}(\mathbf{x}+\mathbf{w})_{1}- \\
\\
d u \frac{\partial L_{0}(\mathbf{x}+\mathbf{w})_{1}}{\partial x}-d v \frac{\partial L_{0}(\mathbf{x}+\mathbf{w})_{1}}{\partial y}-d w \frac{\partial L_{0}(\mathbf{x}+\mathbf{w})_{1}}{\partial z}
\end{aligned}
$$

A particular 1D cartesian Hermite coefficient can be obtained with the inner product between the signal located by the Gaussian window and the corresponding Hermite polynomial as follows [29]:

$$
L_{k}=\left\langle L(x), H_{k}\left(\frac{x}{\sigma}\right)\right\rangle
$$

Therefore, the spatial derivatives of the Hermite coefficients can be expressed as:

$$
L_{k}=\frac{\partial^{k} L(x)}{\partial^{k} x}
$$


for example, we can get the following simplified derivatives for $x$ :

$$
\begin{gathered}
L_{100}(\mathbf{x}+\mathbf{w})=L_{100}(\mathbf{x})_{\mathbf{w}}=\frac{\partial L_{000}(\mathbf{x}+\mathbf{w})_{1}}{\partial x} \\
l_{n, \theta, \phi,(m+1)}(\mathbf{x})_{\mathbf{w}}=\frac{\partial l_{n, \theta, \phi}(\mathbf{x}+\mathbf{w})_{1}}{\partial x}
\end{gathered}
$$

also, we can define the temporal differences as:

$$
\begin{gathered}
L_{0}(\mathbf{x})_{t}=L_{0}(\mathbf{x}+\mathbf{w})_{1}-L_{0}(\mathbf{x})_{0} \\
l_{n, \theta, \phi}(\mathbf{x})_{t}=l_{n, \theta, \phi}(\mathbf{x})_{1}-l_{n, \theta, \phi}(\mathbf{x}+\mathbf{w})_{0}
\end{gathered}
$$

then (17) can be written as

$$
L_{0}(\mathbf{x})_{0}-L_{0}(\mathbf{x}+\mathbf{w}+d \mathbf{w})_{1} \approx-\left[L_{0}(\mathbf{x})_{t}+d u L_{100}(\mathbf{x})_{\mathbf{w}}+d v L_{010}(\mathbf{x})_{\mathbf{w}}+d w L_{001}(\mathbf{x})_{\mathbf{w}}\right]
$$

Finally, we can redefine the 3D Horn-Hermite optical flow (HOF3D) functional from Equation (16) as:

$$
\begin{aligned}
E(\mathbf{w}) & =\int_{V}\left(-\left[L_{0}(\mathbf{x})_{t}+d u L_{100}(\mathbf{x})_{\mathbf{w}}+d v L_{010}(\mathbf{x})_{\mathbf{w}}+d w L_{001}(\mathbf{x})_{\mathbf{w}}\right]^{2}\right. \\
& -\gamma \sum_{n=1}^{N}\left[l_{n, \theta, \phi}(\mathbf{x})_{t}+d u l_{n, \theta, \phi,(m+1)}(\mathbf{x})_{\mathbf{w}}+d v l_{n, \theta, \phi,(n+1)}(\mathbf{x})_{\mathbf{w}}+d w l_{n, \theta, \phi,(l+1)}(\mathbf{x})_{\mathbf{w}}\right]^{2} \\
& \left.+\alpha|\nabla(\mathbf{w}+d \mathbf{w})|^{2}\right) d \mathbf{x}
\end{aligned}
$$

Minimizing $E(\mathbf{w})$ with respect to $u, v$ and $w$ we obtain the following equation system:

$$
\begin{aligned}
& -2\left(L_{0}(\mathbf{x})_{t}+d u L_{100}(\mathbf{x})_{\mathbf{w}}+d v L_{010}(\mathbf{x})_{\mathbf{w}}+d w L_{001}(\mathbf{x})_{\mathbf{w}}\right) L_{100}(\mathbf{x})_{\mathbf{w}} \\
& -2 \gamma \sum_{n=1}^{N}\left(l_{n, \theta, \phi}(\mathbf{x})_{t}+d u l_{n, \theta, \phi,(m+1)}(\mathbf{x})_{\mathbf{w}}+d v l_{n, \theta, \phi,(n+1)}(\mathbf{x})_{\mathbf{w}}+d w l_{n, \theta, \phi,(l+1)}(\mathbf{x})_{\mathbf{w}}\right) l_{n, \theta, \phi,(m+1)}(\mathbf{x})_{\mathbf{w}} \\
& +2 \alpha|\nabla(u+d u)|=0 \\
& -2\left(L_{0}(\mathbf{x})_{t}+d u L_{100}(\mathbf{x})_{\mathbf{w}}+d v L_{010}(\mathbf{x})_{\mathbf{w}}+d w L_{001}(\mathbf{x})_{\mathbf{w}}\right) L_{010}(\mathbf{x})_{\mathbf{w}} \\
& -2 \gamma \sum_{n=1}^{N}\left(l_{n, \theta, \phi}(\mathbf{x})_{t}+d u l_{n, \theta, \phi,(m+1)}(\mathbf{x})_{\mathbf{w}}+d v l_{n, \theta, \phi,(n+1)}(\mathbf{x})_{\mathbf{w}}+d w l_{n, \theta, \phi,(l+1)}(\mathbf{x})_{\mathbf{w}}\right) l_{n, \theta, \phi,(n+1)}(\mathbf{x})_{\mathbf{w}} \\
& +2 \alpha|\nabla(v+d v)|=0 \\
& -2\left(L_{0}(\mathbf{x})_{t}+d u L_{100}(\mathbf{x})_{\mathbf{w}}+d v L_{010}(\mathbf{x})_{\mathbf{w}}+d w L_{001}(\mathbf{x})_{\mathbf{w}}\right) L_{001}(\mathbf{x})_{\mathbf{w}} \\
& -2 \gamma \sum_{n=1}^{N}\left(l_{n, \theta, \phi}(\mathbf{x})_{t}+d u l_{n, \theta, \phi,(m+1)}(\mathbf{x})_{\mathbf{w}}+d v l_{n, \theta, \phi, \phi}(n+1)(\mathbf{x})_{\mathbf{w}}+d w l_{n, \theta, \phi,(l+1)}(\mathbf{x})_{\mathbf{w}}\right) l_{n, \theta, \phi,(l+1)}(\mathbf{x})_{\mathbf{w}} \\
& +2 \alpha|\nabla(w+d w)|=0
\end{aligned}
$$

Rewriting the equation system of (26), (27) and (28) in matrix form we get:

$$
\left[\begin{array}{lll}
A_{1} & A_{2} & A_{3} \\
A_{4} & A_{5} & A_{6} \\
A_{7} & A_{8} & A_{9}
\end{array}\right]\left[\begin{array}{l}
d u \\
d v \\
d w
\end{array}\right]=\left[\begin{array}{l}
b_{1} \\
b_{2} \\
b_{3}
\end{array}\right]
$$

where

$$
\begin{aligned}
& A_{1}=L_{100}^{2}(\mathbf{x})_{\mathbf{w}}+\gamma \sum_{n=1}^{N} l_{n, \theta, \phi,(m+1)}^{2}(\mathbf{x})_{\mathbf{w}} \\
& A_{2}=L_{100}(\mathbf{x})_{\mathbf{w}} L_{010}(\mathbf{x})_{\mathbf{w}}+\gamma \sum_{n=1}^{N} l_{n, \theta, \phi,(m+1)}(\mathbf{x})_{\mathbf{w}} \cdot l_{n, \theta, \phi,(n+1)}(\mathbf{x})_{\mathbf{w}}
\end{aligned}
$$




$$
\begin{aligned}
& A_{3}=L_{100}(\mathbf{x})_{\mathbf{w}} L_{001}(\mathbf{x})_{\mathbf{w}}+\gamma \sum_{n=1}^{N} l_{n, \theta, \phi,(n+1)}(\mathbf{x})_{\mathbf{w}} \cdot l_{n, \theta, \phi,(l+1)}(\mathbf{x})_{\mathbf{w}} \\
& b_{1}=L_{0}(\mathbf{x})_{t} L_{100}(\mathbf{x})_{\mathbf{w}}+\gamma \sum_{n=1}^{N} l_{n, \theta, \phi}(\mathbf{x})_{t} \cdot l_{n, \theta, \phi,(m+1)}(\mathbf{x})_{\mathbf{w}}-\alpha|\nabla(u+d u)| \\
& A_{4}=L_{010}(\mathbf{x})_{\mathbf{w}} L_{100}(\mathbf{x})_{\mathbf{w}}+\gamma \sum_{n=1}^{N} l_{n, \theta, \phi,(n+1)}(\mathbf{x})_{\mathbf{w}} \cdot l_{n, \theta, \phi,(m+1)}(\mathbf{x})_{\mathbf{w}} \\
& A_{5}=L_{010}^{2}(\mathbf{x})_{\mathbf{w}}+\gamma \sum_{n=1}^{N} l_{n, \theta, \phi,(n+1)}^{2}(\mathbf{x})_{\mathbf{w}} \\
& A_{6}=L_{010}(\mathbf{x})_{\mathbf{w}} L_{001}(\mathbf{x})_{\mathbf{w}}+\gamma \sum_{n=1}^{N} l_{n, \theta, \phi,(m+1)}(\mathbf{x})_{\mathbf{w}} \cdot l_{n, \theta, \phi,(l+1)}(\mathbf{x})_{\mathbf{w}} \\
& b_{2}=L_{0}(\mathbf{x})_{t} L_{010}(\mathbf{x})_{\mathbf{w}}+\gamma \sum_{n=1}^{N} l_{n, \theta, \phi}(\mathbf{x})_{t} \cdot l_{n, \theta, \phi,(n+1)}(\mathbf{x})_{\mathbf{w}}-\alpha|\nabla(v+d v)| \\
& A_{7}=L_{001}(\mathbf{x})_{\mathbf{w}} L_{100}(\mathbf{x})_{\mathbf{w}}+\gamma \sum_{n=1}^{N} l_{n, \theta, \phi,(l+1)}(\mathbf{x})_{\mathbf{w}} \cdot l_{n, \theta, \phi,(m+1)}(\mathbf{x})_{\mathbf{w}} \\
& A_{8}=L_{001}(\mathbf{x})_{\mathbf{w}} L_{010}(\mathbf{x})_{\mathbf{w}}+\gamma \sum_{n=1}^{N} l_{n, \theta, \phi,(l+1)}(\mathbf{x})_{\mathbf{w}} \cdot l_{n, \theta, \phi,(n+1)}(\mathbf{x})_{\mathbf{w}} \\
& A_{9}=L_{001}^{2}(\mathbf{x})_{\mathbf{w}}+\gamma \sum_{n=1}^{N} l_{n, \theta, \phi,(l+1)}^{2}(\mathbf{x})_{\mathbf{w}} \\
& b_{3}=L_{0}(\mathbf{x})_{t} L_{001}(\mathbf{x})_{\mathbf{w}}+\gamma \sum_{n=1}^{N} l_{n, \theta, \phi}(\mathbf{x})_{t} \cdot l_{n, \theta, \phi,(l+1)}(\mathbf{x})_{\mathbf{w}}-\alpha|\nabla(w+d w)|
\end{aligned}
$$

Finally, in each lower-resolution level, the increment $d \mathbf{w}$ is estimated and, $\mathbf{w}$ is updated in the next high-resolution level.

In this work, we take advantage of the characteristics of the Hermite multiresolution transform, which makes it possible to improve spatial frequency locations and facilitate the analysis of local orientations at different scales [59,60]. Likewise, the HOF3D functional of Equation (25) can calculate small displacements $d u, d v, d w$ and propagate the solution to higher resolution levels. For each resolution level, an iterative method for solving linear equations was carried out.

\section{Materials and Overview of the Method}

\subsection{Dataset Description}

The dataset used in this work consists of two cardiac computed tomography studies $(3 D+t)$. The CT volumes were obtained in a 16-slice tomograph (at $120 \mathrm{kVp} @ 900 \mathrm{~mA}$ ) built with 128 detectors. The dimensions of each volume are $512 \times 512 \times 10$ at 12 bits per pixel. The clinical protocol starts by injected a contrast agent to the patient and the study is carried out in synchrony with the electrocardiogram (ECG) signal. A cardiac CT volume used is shown in Figure 6.

It should be noted that the acquisition of cardiac images are performed in connection with the electrocardiogram and are acquired with the patient in respiratory apnea to avoid artifice by movement.

\subsection{Ethical Approval}

The Research Committee of Engineering Faculty of Universidad Nacional Autonóma de México approved this research protocol. This study was conducted in accordance with the Declaration of Helsinki. 


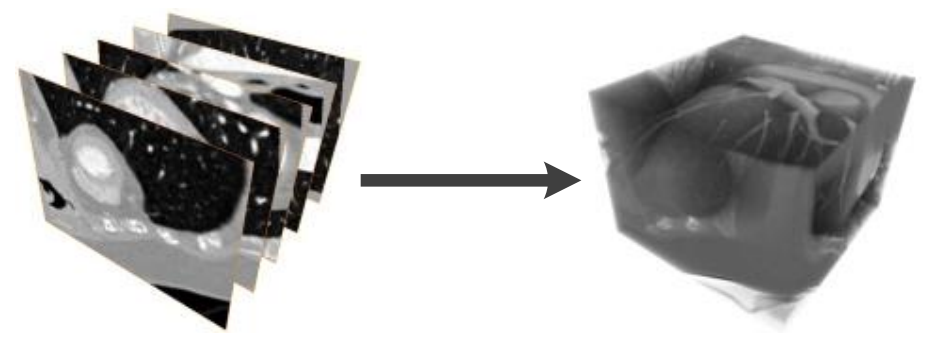

Figure 6. Cardiac CT images, slices and volume.

\subsection{Overview of the Method}

In Figure 7 we show an overview of our proposal. First, we have the cardiac volume slices, belonging to two consecutive steps of the cardiac cycle considered, which make up volume 1 and volume 2 respectively; for each volume, we obtain a multiresolution expansion related to the coefficients of the steered Hermite transform. Such coefficients are used to carry out the calculation of the optical flow within the mentioned HOF3D approach. Once the vector field belonging to the optical flow over the whole cardiac volume was obtained, we used the portion of the volume related to the segmented left ventricle to finally obtain only the masked vectors with this part of the cardiac volume.

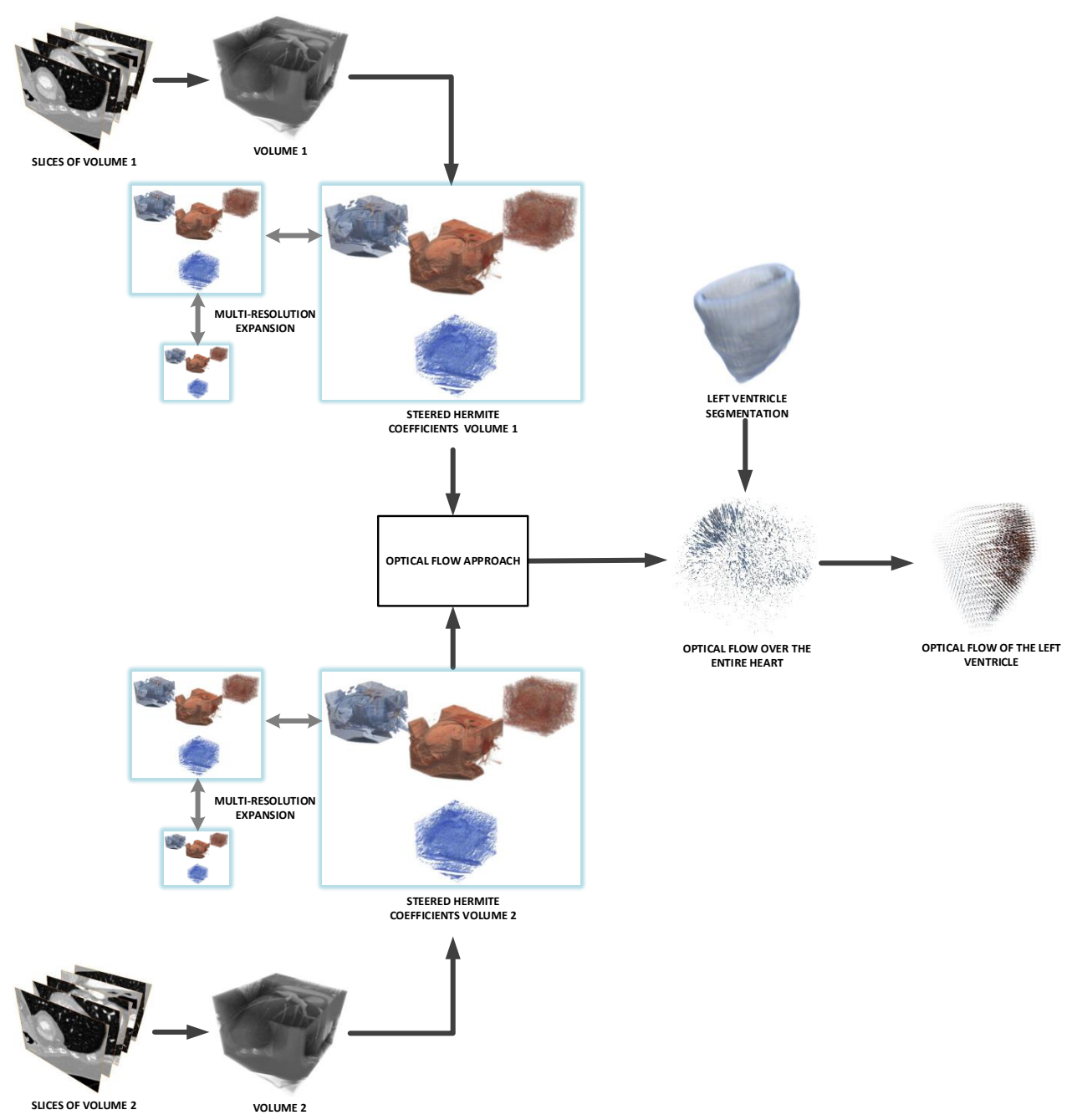

Figure 7. Procedure to implement the HOF3D approach. 


\section{Experiments and Results}

This section presents the results of the estimation of the optical flow with the proposed method. In our previous work [49], we use some synthetic volumes to check the expected results, in this work, dozens of cardiac volumes corresponding to medical CT images, as well as their respective segmentation of the left ventricle, were used.

The section is divided into two stages, a validation stage and a stage of 3D optical flow results of the left ventricle. In the first stage, we performed a validation of our approach, where the optimal parameters both in the Hermite transform and the 3D optical flow proposal were determined. Then, the optical flow results in 2D were compared with a set of images ground-truth and a pair of algorithms of optical flow. The optical flow results in 3D were compared with the modified and multiresolution method of Horn and Schunck [20]. Next, an analysis of robustness to noise was performed. In the second stage, the 3D optical flow results in the left ventricle, which was previously segmented, are shown and the corresponding errors of interpolation are evaluated.

The results obtained on a PC Intel(R) Core(TM) i7-4710HQ CPU running at $2.50 \mathrm{GHz}$ with $16 \mathrm{~GB}$ of RAM have an algorithm time-consuming of $4.8 \mathrm{~h}$ on 4 cores, nevertheless, this can be reduced to an average of $4.5 \mathrm{~min}$ with parallel computing and additional cores. The optical flow in our method has good scalability, close to linear speedup, which allows us to significantly reduce processing time. The results concerning processing time are consistent with those reported in [61]. They tested two differential algorithms, Lucas-Kanade and Horn-Schunck in $3 D+t$, as we have also done.

\subsection{Validation}

In absence of a 3D motion ground-truth in CT images, which is used to evaluate the accuracy of the optical flow estimation, we validate our proposal in two different ways, first, by calculating a forward reconstruction using the volume $L(\mathbf{x}, t)$ at time $t$ and the 3D optical flow obtained and second, comparing our 2D approach with other methods and using a 2D dataset with known ground-truth. In both cases, we used the interpolation error, which is defined as the root mean-square (RMS) difference between the known volume $L(\mathbf{x}, t+1)$ at time $t+1$ and the reconstructed volume $L_{G T}(\mathbf{x}, t+1)$, is calculated $[6,19]$ as we showed in the Equation (30):

$$
I E_{3 D}=\left[\frac{1}{M} \sum_{\mathbf{x}}\left(L(\mathbf{x}, t+1)-L_{G T}(\mathbf{x}, t+1)\right)^{2}\right]^{\frac{1}{2}}
$$

where $M$ is the number of voxels.

We also computed a second measure of interpolation performance, the normalized interpolation error between an interpolated volume $L(\mathbf{x}, t+1)$ and a ground-truth volume $L_{G T}(\mathbf{x}, t+1)$, which is given as in [62]:

$$
N E_{3 D}=\left[\frac{1}{M} \sum_{\mathbf{x}} \frac{\left(L(\mathbf{x}, t+1)-L_{G T}(\mathbf{x}, t+1)\right)^{2}}{\left\|\nabla L_{G T}(\mathbf{x}, t+1)\right\|^{2}+\varepsilon}\right]^{\frac{1}{2}}
$$

that represents a gradient-normalized RMS error, where $\varepsilon$ is a scaling constant (e.g., $\varepsilon=1$ ).

The interpolation errors are useful to know how good the calculation of the optical flow is when there is no available ground truth flow, the normalized interpolation error has the additional advantage of being normalized with respect to the magnitudes of the intensity changes that the volume of the reference.

\subsubsection{Hermite Transform Parameter Tuning}

Although the constants, values and weight parameters are difficult to select, in Section 2, we present which are the suitable values to the cubic window and in consequence, the maximum 
expansion order $N$ of the Hermite transform, thus, experimentally found that we achieved a good estimation of optical flow results $\left(N E_{3 D}<0.1\right)$ and avoiding blur artifacts for our dataset with: a cubic window of $5 \times 5 \times 5$ pixels, i.e., a maximum expansion order of $N=4$ for the SHT and 5 levels of multiresolution decomposition for the SHT. Below these values, we would obtain errors 2.5 to 3 times larger than those reported. It should be noted that this strategy allows us to handle large displacements, which occur from one step to another in a cardiac cycle. On the other hand, the number of iterations greater than 50 is the one that gives us the required numerical convergence according to our tests.

\subsubsection{Optical Flow Parameter Sensitivity Analysis}

As a first experiment, we perform a parameter sensitivity analysis to find the best values. Weight parameter $\gamma$ of the HOF3D functional Equation (16) is used to weigh the contribution of the high order Hermite coefficients in those regions where the intensity does not remain constant from one volume to another. On the other hand, the softness parameter $\alpha$ can help recover the motion information from their neighbors in those regions where the gradient is zero, e.g., intensity homogeneous regions. It is carried out through averages from structures with high frequencies, e.g., edges and textures. Large values of $\alpha$ give us a smoother flow but this is relatively less important at locations with high image gradients than elsewhere.

For determining the values of the smoothness weight $\alpha$ and the weight parameter $\gamma$, first we compute the 3D optical flow over the cardiac CT sequences and then we analyze the behavior of the Interpolation Error (IE) and Normalized Interpolation Error (NE) metrics.

From Figure 8, the curves show that the best results for IE and NE are for $\alpha \geq 10$ and $\gamma \geq 100$ (bottom of the mesh).

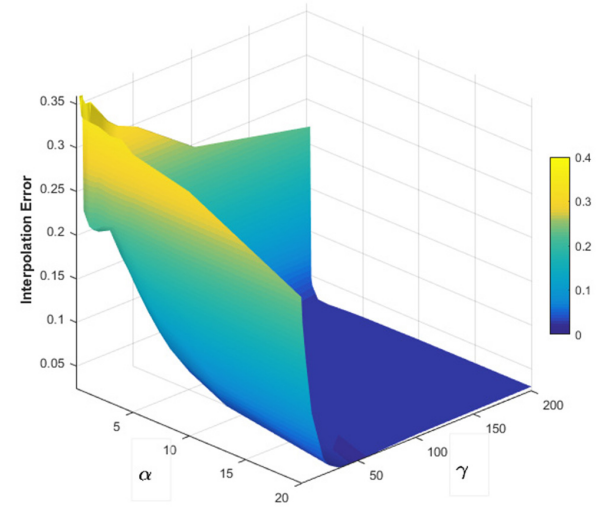

(a)

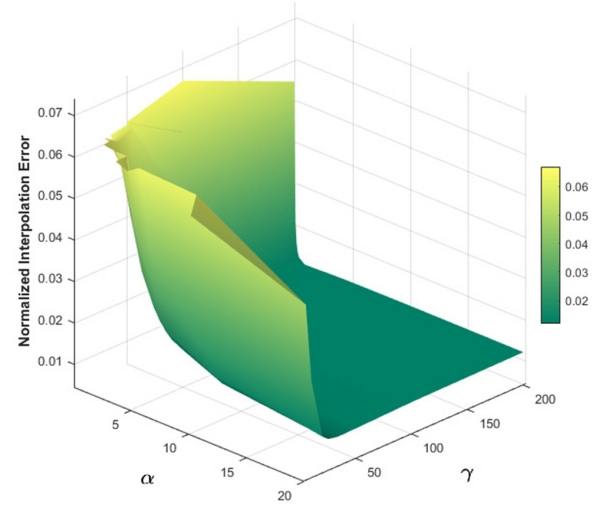

(b)

Figure 8. Interpolation Error (a) and Normalized Interpolation Error (b), for parameter sensitivity analysis.

\subsubsection{D Interpolation Errors}

As we mentioned before, because we do not have a set of 3D optical flow to compare our results, as second experiment, we evaluate the performance of our 2D proposal, through a collection of well-known images. These images and their respective ground-truth optical flows can be found through [63], which still have great use and relevance today. They defined sequences with non-rigid movements where the optical flow was determined following a hidden fluorescent texture.

Table 2 shows the calculation of the interpolation error (Equation (30)) but in 2D. We choose a set of five data. HOF2D is the Horn-Hermite optical flow in 2D approach. We compare our HOF2D algorithm, along with another pair of algorithms and the ground-truth flows provided in [63]. The parameters 
used for HOF2D are the same as those described in Sections 5.1.1 and 5.1.2, $N=4,5$ levels of multiresolution decomposition for the SHT in 2D, $\alpha \geq 10$ and $\gamma \geq 100$, except that the necessary iterations, which can range from 20, to provide the best results. The best results are highlighted in bold and, although our approach is not always the best, it is close to the best results in each case.

Table 2. Interpolation Error Calculation.

\begin{tabular}{ccccc}
\hline Ground Truth Images & Ground Truth Flow & Horn-Schunck [64] & Farnebäck [64] & HOF2D \\
\hline dimetrodon & 2.641 & 8.589 & 3.127 & $\mathbf{2 . 8 6 5}$ \\
groove2 & 10.439 & 23.492 & 8.831 & $\mathbf{1 0 . 3 5 3}$ \\
groove3 & 19.401 & 32.351 & $\mathbf{1 5 . 7 0 3}$ & 17.460 \\
urban3 & 9.870 & 17.727 & 9.489 & $\mathbf{8 . 1 2 2}$ \\
venus & 8.813 & 20.659 & $\mathbf{5 . 8 4 7}$ & 8.835 \\
\hline
\end{tabular}

With the same set of data and algorithms as Table 2, Table 3 presents the calculation of the normalized interpolation error in 2D based on (Equation (31)). The best performances are highlighted in bold and most of them are in the HOF2D column. We must remember that the normalized interpolation error is is a weighted RMS average of the pixels, wich use the image gradient as a weight factor. The normalized interpolation error compensates for the difference between the interpolation errors and the flow obtained because it gives less weight to the discontinuous regions and more weight to the regions without texture.

Table 3. Normal Interpolation Error Calculation.

\begin{tabular}{ccccc}
\hline Ground Truth Images & Ground Truth Flow & Horn-Schunck [64] & Farnebäck [64] & HOF2D \\
\hline dimetrodon & 0.207 & 0.546 & 0.382 & $\mathbf{0 . 2 7 0}$ \\
groove2 & 0.418 & 0.860 & 0.385 & $\mathbf{0 . 3 2 9}$ \\
groove3 & 0.990 & 1.622 & 0.626 & $\mathbf{0 . 5 3 2}$ \\
urban3 & 2.325 & 2.452 & 1.342 & $\mathbf{0 . 7 0 0}$ \\
venus & 0.801 & 1.376 & 0.434 & $\mathbf{0 . 3 4 8}$ \\
\hline
\end{tabular}

\subsubsection{D Interpolation Errors}

To evaluate the accuracy of the HOF3D method, we compared it with the 3D variant of the method of Sun et al. [20] We calculated the corresponding interpolation errors (IE and NE) using both proposals. Figure 9 presents a diagram of the steps to calculate the interpolation errors.

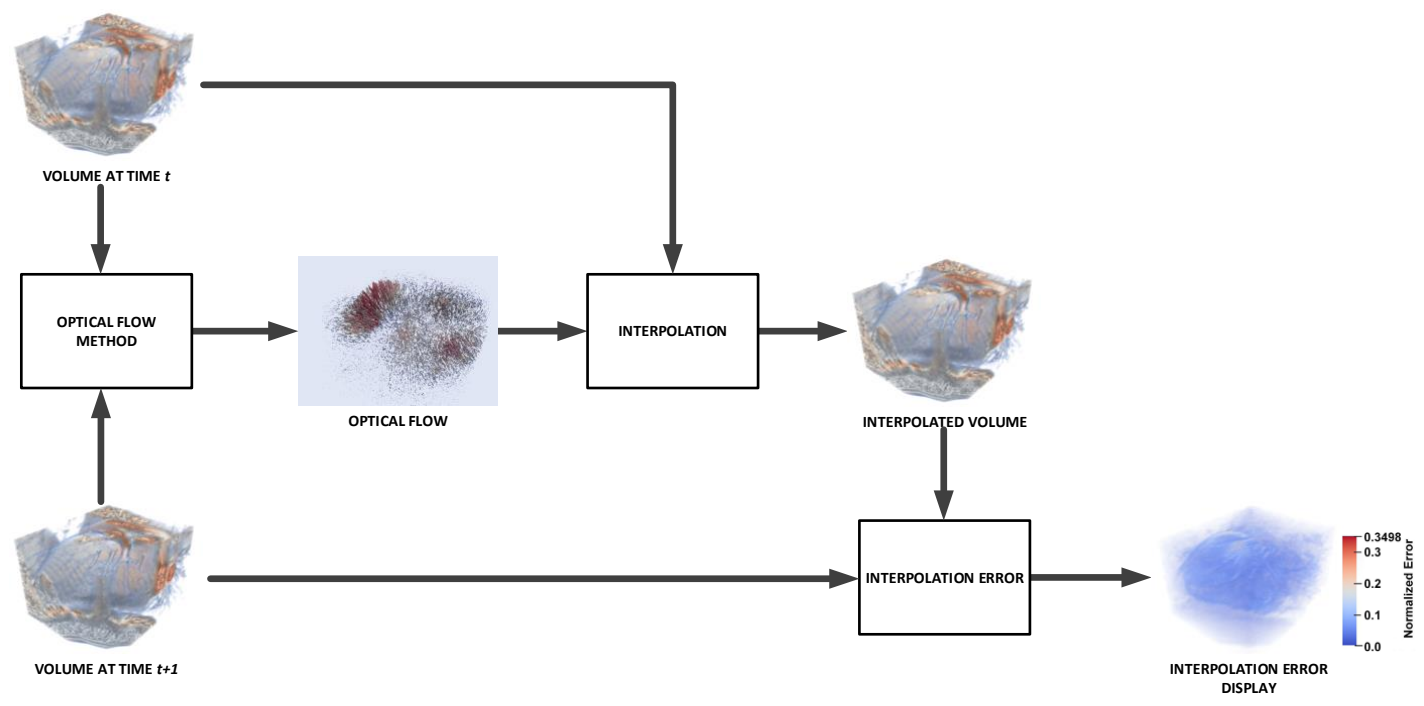

Figure 9. Steps to calculate and visualize the Interpolation Error. 
Figure 10 shows a cardiac CT volume where we can observe the original volume (Figure 10a), the interpolated volume (Figure 10b), the difference between the original volume and its interpolated result (Figure 10c) using the 3D variant of the method of Sun et al. [20]. The results were compared with a modified version of the Sun method in 3D and for different noise levels. Both algorithms used were optimized and the evaluation of the results was carried out by means of a forward reconstruction, from the volume at time $t$ to time $t+1$, through the $3 \mathrm{D}$ optical flow obtained. The interpolation error display is a visualization of the terms within the summation in Equation (31).

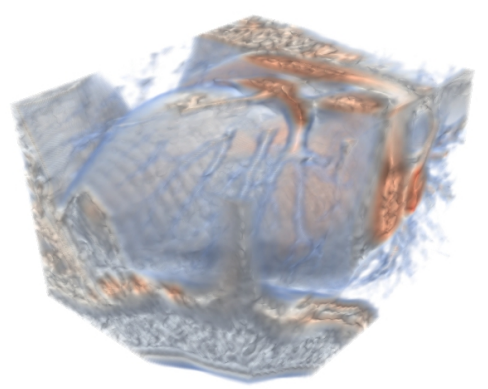

(a) Original Volume

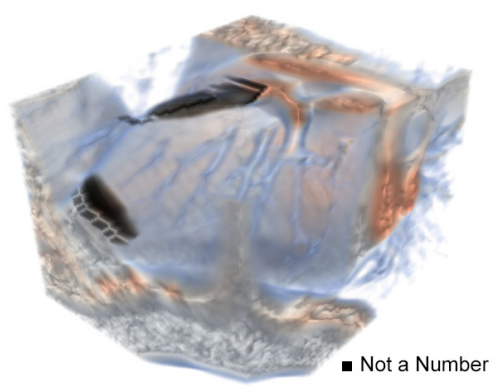

(b) Volume Interpolated using the Horn and Schunck method

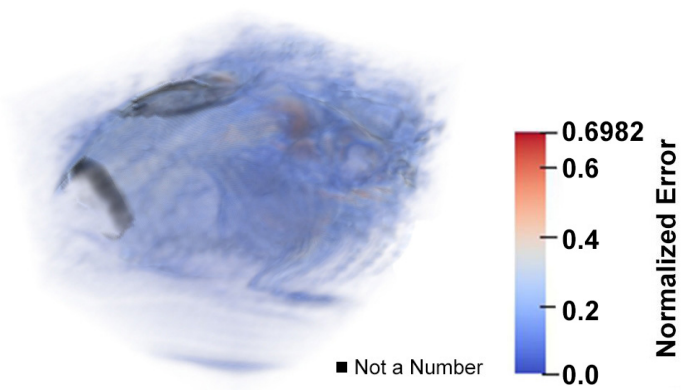

(c) Error between Original and Interpolated Volumes using the Horn and Schunck method

Figure 10. A cardiac CT volume showing the original volume, the interpolated volume using the 3D variant of the method of Sun et al. [20] and the error between the original volume and its interpolated result.

On the other hand, in order to compare our method, in Figure 11 we show the same cardiac CT volume as in Figure 10 where, again, we can observe the original volume (Figure 11a), the interpolated volume (Figure 11b), the difference between the original volume and its interpolated result (Figure 11c) using our HOF3D method.

In Figure 12, we show the interpolation errors obtained using the Sun et al. [20] and the HOF3D methods through the whole cardiac cycle (0\% to $90 \%)$ for two CT sequences. In both sequences, we can observe the beginning of the increase in the interpolation error from $20 \%$ to $30 \%$, when the contraction movement occurs and from $50 \%$ to $60 \%$ of the cardiac cycle, in full dilation movement. This is where we have a couple of cardiac movements of greater magnitude.

We can observe in the plots of Figure 12, both for the interpolation error and the normalized interpolation error, that even in each of the stages of the complete cardiac cycle, the HOF3D method gives better results and lower errors are obtained. 


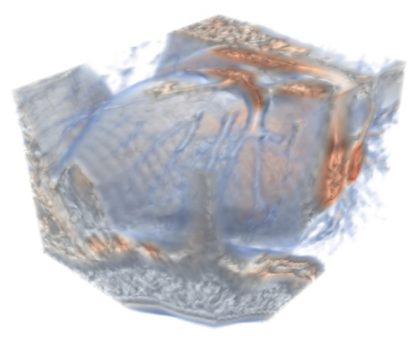

(a) Original Volume

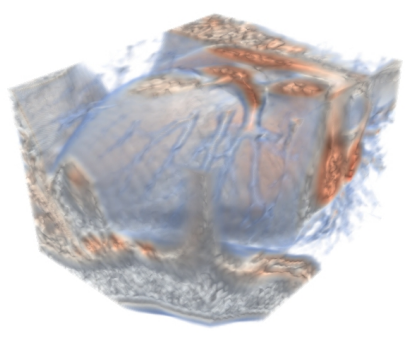

(b) Volume Interpolated using the HOF3D method

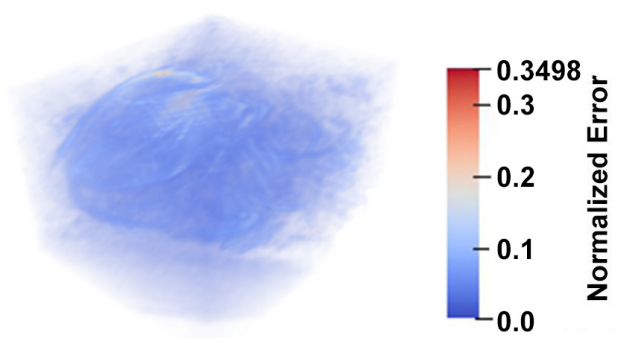

(c) Error between Original and Interpolated Volumes using HOF3D method

Figure 11. A cardiac CT volume showing the original volume, the Interpolated Volume using the HOF3D method and the error between the original volume and its interpolated result.

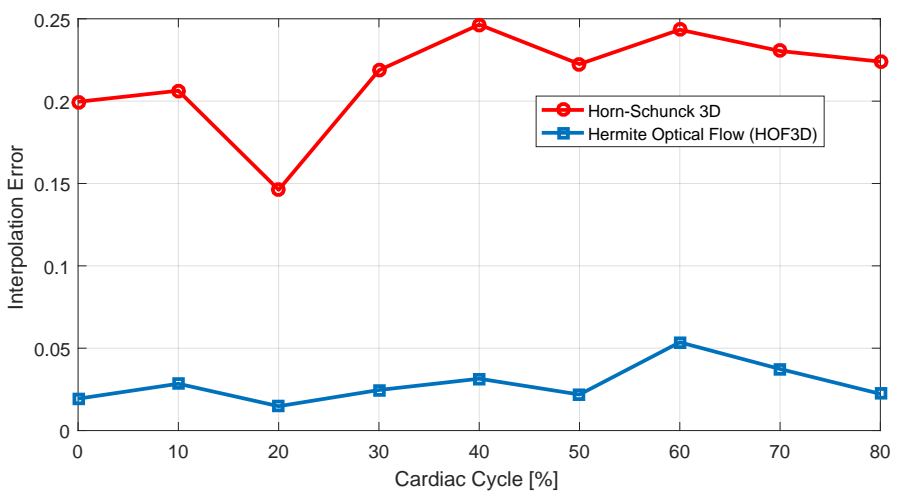

(a) Interpolation Error

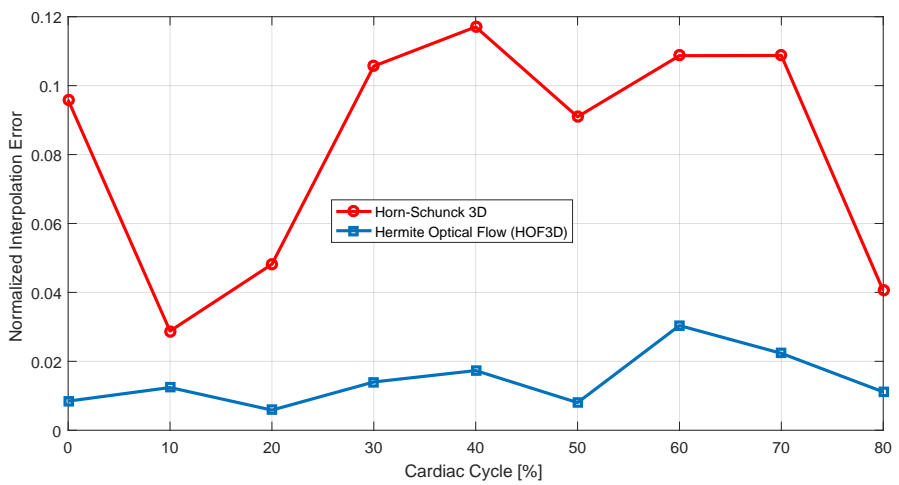

(b) Normalized Interpolation Error

Figure 12. Interpolation Error and Normalized Interpolation Error. For the 3D Horn-Schunck (red dashed line) and Hermite Optical Flow in 3D (blue solid line) methods. From sequences of cardiac CT volumes. 


\subsubsection{Robustness to Noise}

As final experiment, we carried out an analysis of robustness to noise of the proposed method. For this, we added Gaussian noise, with different standard deviations $\left(\sigma_{n}=0,5,10,15,20,30\right)$ and zero mean, to the cardiac volumes. In Figure 13 we can see one of the volumes used for the test, with three different values of $\sigma_{n}$.

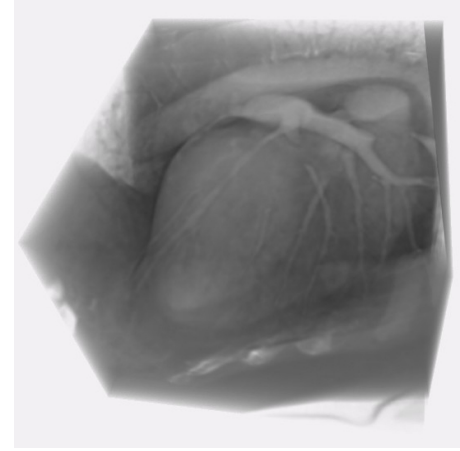

(a) Volume without noise

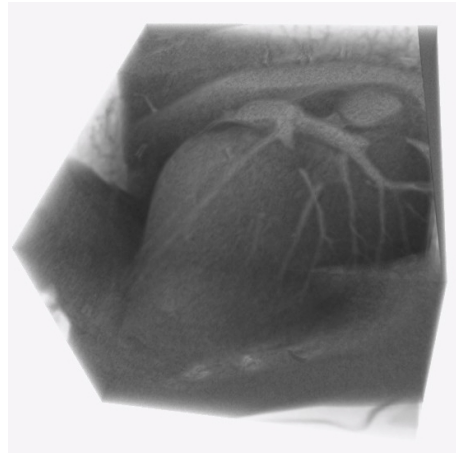

(b) Volume with noise, $\left(\sigma_{n}=10\right)$

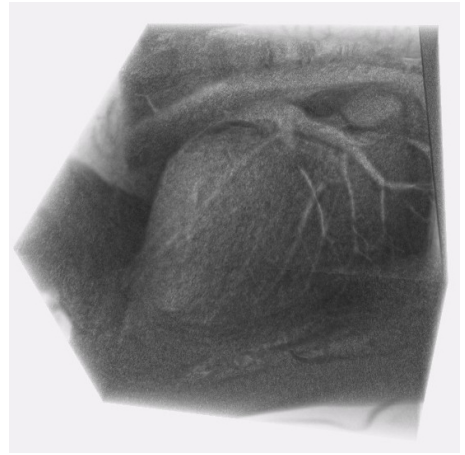

(c) Volume with noise, $\left(\sigma_{n}=30\right)$

Figure 13. Volume with pseudo-random noise.

Table 4 shows the interpolation error and the normalized interpolation error for the noise levels given for the HOF3D method, using optimized parameters $\alpha=10, \gamma=100$ and $N=4$. We can observe that although the standard deviation of the introduced noise grows, the interpolation error and the normalized interpolation error remain small, this is because the coefficient of order 0 of the Hermite transform $L_{000}(\mathbf{x})$ (Equations (3), (11) and (16)), contains a smoothed version of the original volume and this DC coefficient allows to reduce any component of high-frequency noise, additionally, in our approach, the steered Hermite coefficients use Gaussian derivatives, which incorporate information from neighboring voxels in the structure of cardiac volumes, which makes the proposed algorithm more robust to this type of noise $[13,65]$. By the other hand, it should be noted that although the errors are low, the addition of noise represents an increase in the interpolation error of $71.4 \%$ and for the normalized interpolation error of $80 \%$, comparing one test without noise and the other test with noise of $\sigma_{n}=30$.

Table 4. Interpolation Error and Normalized Interpolation Error computed for a cardiac volume with several standard deviations $\sigma_{n}$ of Gaussian noise.

\begin{tabular}{ccc}
\hline Gaussian Noise $\left(\sigma_{n}\right)$ & Interpolation Error & Normalized Interpolation Error \\
\hline 0 & 0.03190 & 0.01696 \\
5 & 0.03499 & 0.01954 \\
10 & 0.03778 & 0.02168 \\
15 & 0.04295 & 0.02563 \\
20 & 0.04597 & 0.02779 \\
30 & 0.05468 & 0.03387 \\
\hline
\end{tabular}

\subsection{D Optical Flow Results}

In this section, we show the 3D optical flow estimation computed on CT volumes for a whole cardiac cycle. For descriptive purposes, only some representative parts of such a cardiac cycle are shown. In most cases, the display of the magnitudes of the optical flows was exaggerated in order to observe the qualitative characteristics of the movements.

Figure 14 shows the results of a 3D Optical flow of two cardiac CT volumes computed at phases 20-30\% (when a contraction movement occurs) using the HOF3D method. Figure 14a,b show two phases of the cardiac cycle of volume (for better viewing a cut of that volume was made). 
Figure $14 \mathrm{c}, \mathrm{d}$ show the same phases of volume along with the three-dimensional optical flow field. Finally, Figure 14e,f illustrate only the optical flow.

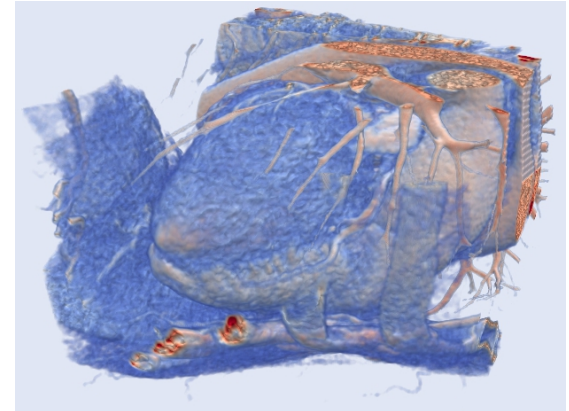

(a) Volume - 20\%

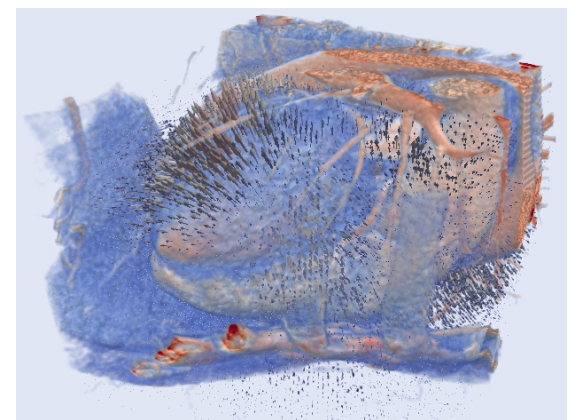

(c) Volume and Optical Flow - 20\%

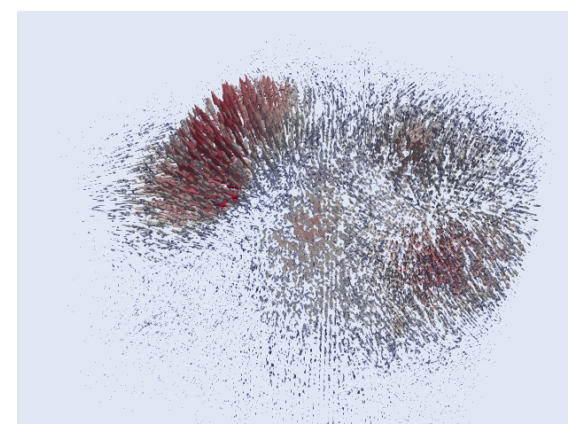

(e) Only Optical Flow - 20\%

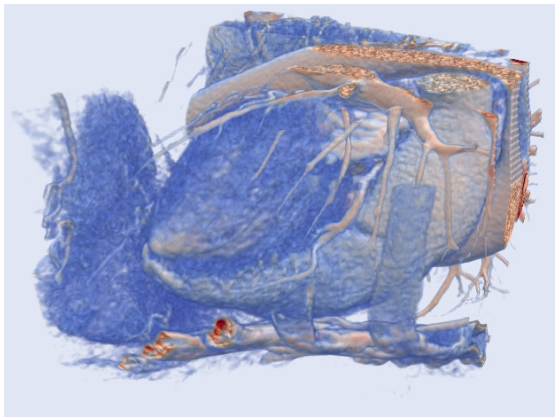

(b) Volume - 30\%

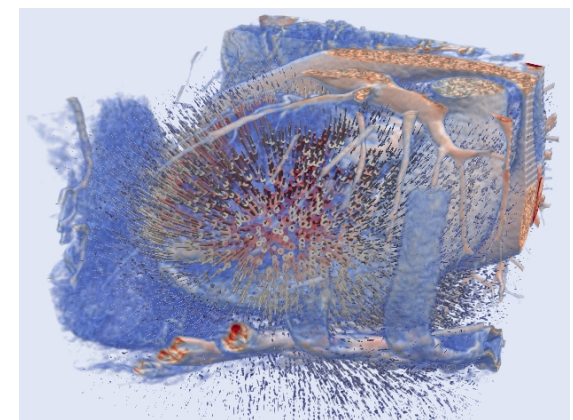

(d) Volume and Optical Flow - 30\%

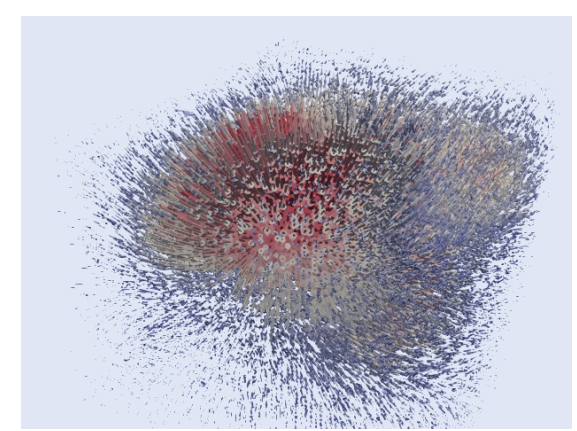

(f) Only Optical Flow - 30\%

Figure 14. Results of 3D Optical Flow of a segmented cardiac CT volume computed at phases 20-30\%.

Figure 15 presents the results of a 3D Optical flow of two cardiac CT volumes computed at phases $50-60 \%$ (when a dilation movement occurs) using the HOF3D method. Figure 15a,b show two phases of the cardiac cycle of volume (for better viewing a cut that volume was made). Figure $15 \mathrm{c}$, d show the same phases of volume along with the three-dimensional optical flow field. Also, Figure 15e,f present only the optical flow. 


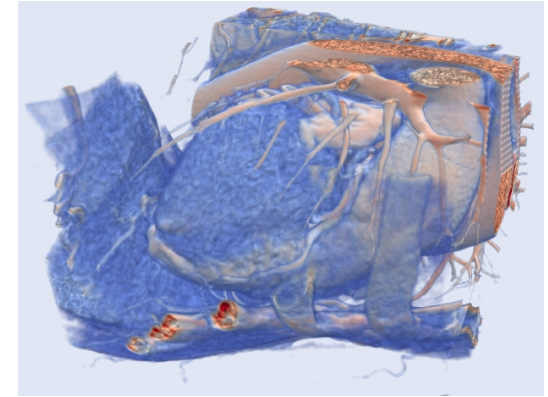

(a) Volume - $50 \%$

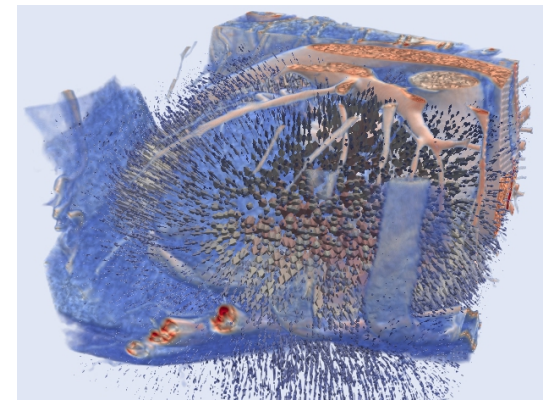

(c) Volume and Optical Flow - 50\%

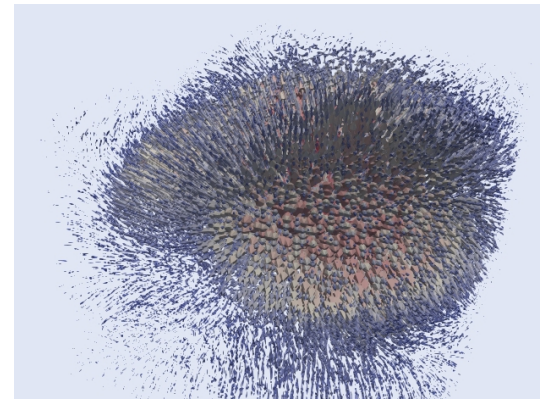

(e) Only Optical Flow - 50\%

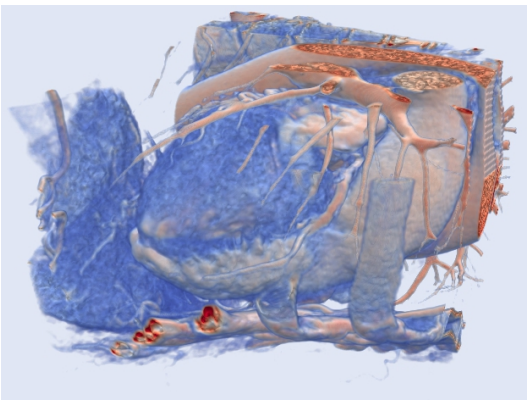

(b) Volume - $60 \%$

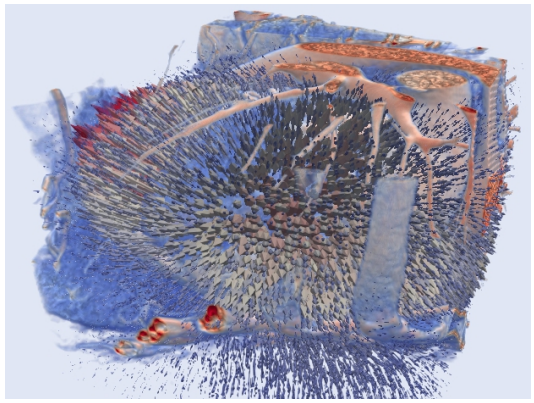

(d) Volume and Optical Flow - 60\%

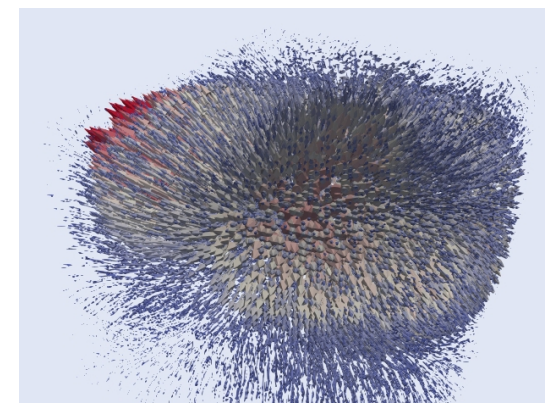

(f) Only Optical Flow - 60\%

Figure 15. Results of 3D Optical Flow of a segmented cardiac CT volume computed at phases 50-60\%.

\subsubsection{D Optical Flow Estimation of the Left Ventricle}

The importance of the study of the left ventricle has been established extensively. The left ventricle adapts, for example, to arterial hypertension and this leads to the development of different geometric patterns [66]. For a better understanding of some diseases, the movement of the left ventricle has been studied during the cardiac cycle in normal subjects and patients with coronary arterial disease, mitral stenosis or atrial septal defect [67]. Works describing the global and local movement have been presented, focusing mainly on the left ventricle [68]. To present the optical flow estimation of the left ventricle, first, a segmentation of it is required. We use the level sets method of Osher and Sethian [69]. This method is a powerful, suitable and flexible approach to segmentation of CT volumes where there aren't well-defined boundaries. The level sets method was applied to the CT volumes using the Seg3D tool [70]. For this tool, a seed volume is used to find similar regions to the original one. Then, the segmented region will be expanded to surrounding pixels that match the statistics of the original seeded area. The spread may also be retracted in some instances if the seeded areas do not match certain criteria (edge weight and threshold range). Until the convergence, the algorithm will be expanded (or contracted) to the segmented region.

Figure 16 shows an example of the segmentations obtained (colored region) in the context of their location within the whole cardiac volume. 


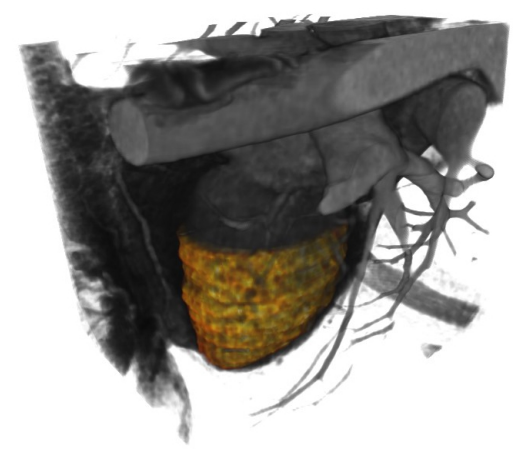

Figure 16. A whole cardiac volume and its left ventricle segmented.

Similarly to the work done in Section 5.1.4, and if we focus on the phase where a contraction movement occurs for the left ventricle, we can observe the interpolation error for a left ventricle segmented showing the original volume in Figure 17a, the interpolated volume using the 3D variant of the method of Sun et al. [20] are in Figure 17b, the error between the original volume and this interpolated result, in Figure 17d. Also, the optical flow is calculated by the HOF3D method. Then, the interpolation of the left ventricle is obtained, which is shown in Figure 17c. The difference between the original volume and that interpolated volume can be observed in Figure 17e.

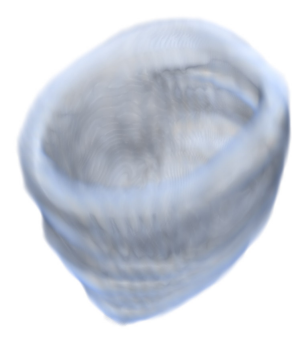

(a) Original LV- $30 \%$
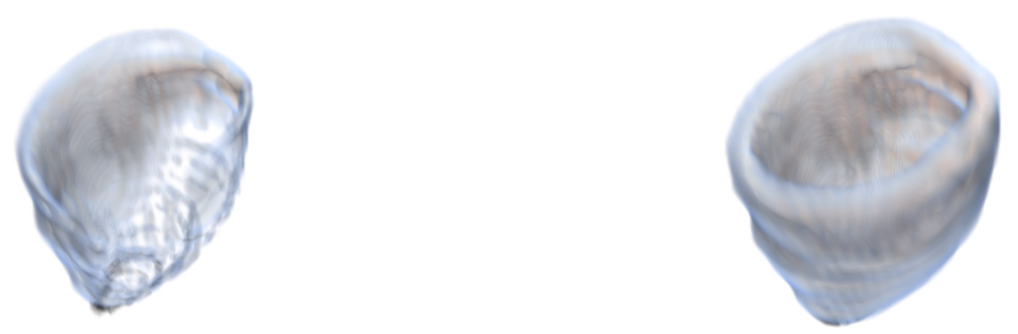

(b) LV Interpolated using the 3D method of Sun et al. [20]

(c) LV Interpolated using the HOF3D method
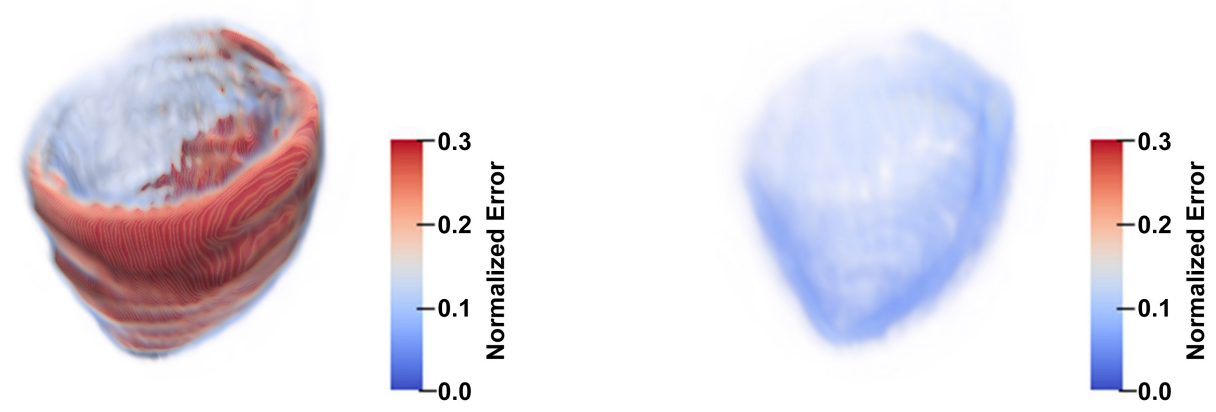

(d) Error using the 3D method of Sun et al. [20]

(e) Error using the HOF3D method

Figure 17. Interpolation Errors for the left ventricle at $30 \%$. 
Another example of interest, where there is more interpolation error, is the $60 \%$ of cardiac phase, it is also when a dilatation movement occurs for the left ventricle. We can observe the interpolation error for a left ventricle segmented for the original volume in Figure 18a, the interpolated volume using the 3D method of Sun et al. [20] in Figure 18b, the error between the original volume and this interpolated result in Figure 18d. Also, the optical flow is calculated by the HOF3D method. Then, the interpolation of the left ventricle is obtained and shown in Figure 18c. The difference between the original volume and that interpolated volume can be observed in Figure 18e.

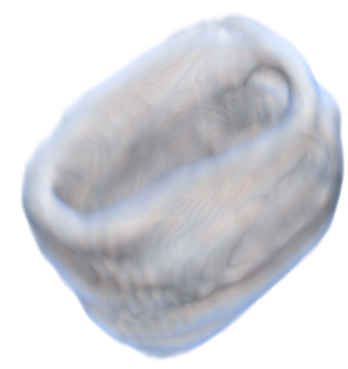

(a) Original LV- $60 \%$

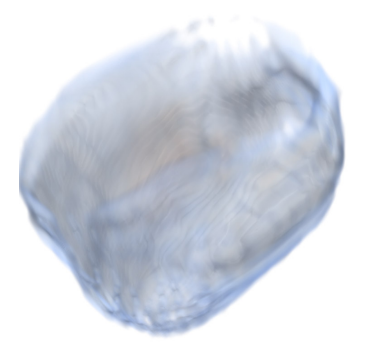

(b)LV Interpolated using the 3D method of Sun et al. [20]

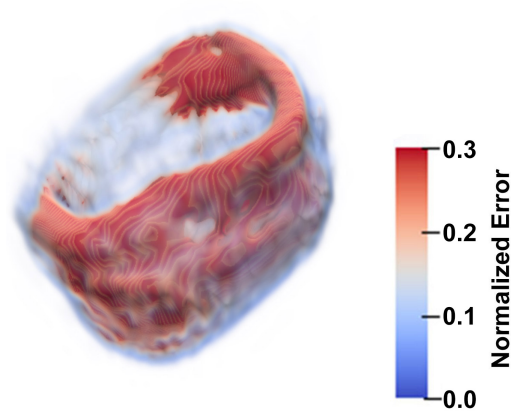

(d) Error using the 3D method of Sun et al. [20]

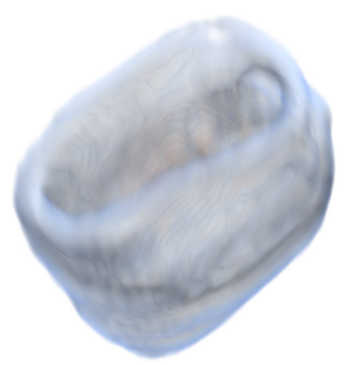

(c) LV Interpolated using the HOF3D

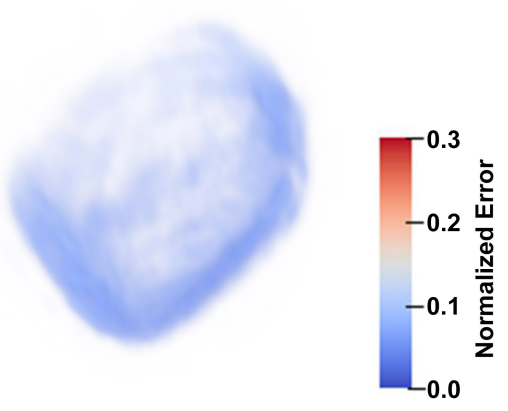

(e) Error using the HOF3D method

Figure 18. Interpolation Errors for the left ventricle at $60 \%$.

Figure 19 contains the results of the normalized interpolation error with the Sun et al. [20] method. In Figure 19a we present a set of volumes from 30\% to $70 \%$ of the cardiac cycle, in Figure 19b, the interpolated volumes corresponding to each stage of the cardiac cycle are shown. Figure 19c graphically displays the normalized interpolation error for the volumes of sections a and $b$ respectively.

Figure 20 contains the results of the normalized interpolation error with the HOF3D method. Figure 20 a presents a set of volumes from $30 \%$ to $70 \%$ of the cardiac cycle, Figure $20 \mathrm{~b}$ shows the interpolated volumes corresponding to each stage of the cardiac cycle. Figure 20c graphically displays the normalized interpolation error for the volumes of sections a and $b$ respectively. 

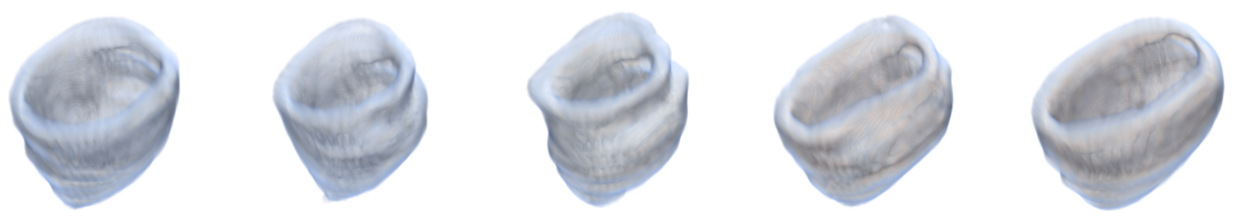

(a) Original Volumes
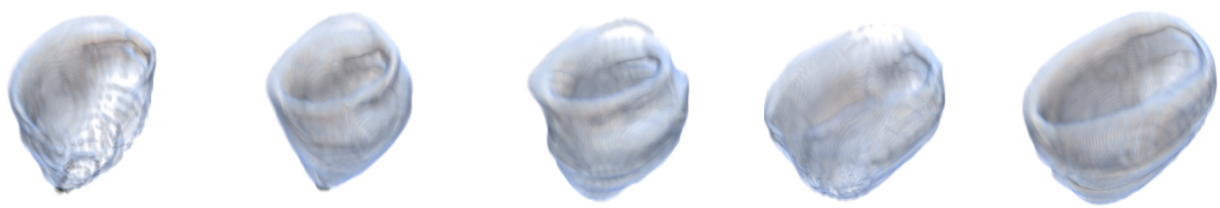

(b) Interpolated Volumes
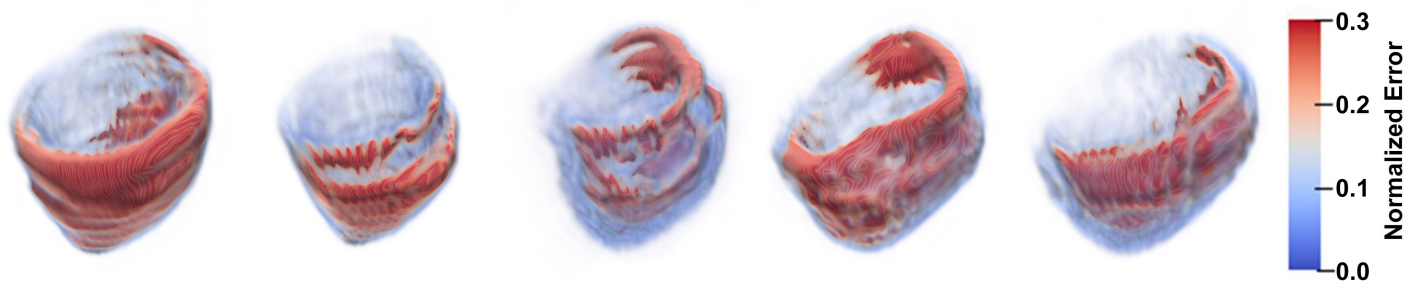

(c) Normalized Interpolation Error

Figure 19. Left Ventricle from $30 \%$ to $70 \%$ of the cardiac cycle and the results with 3D method of Sun et al. [20].
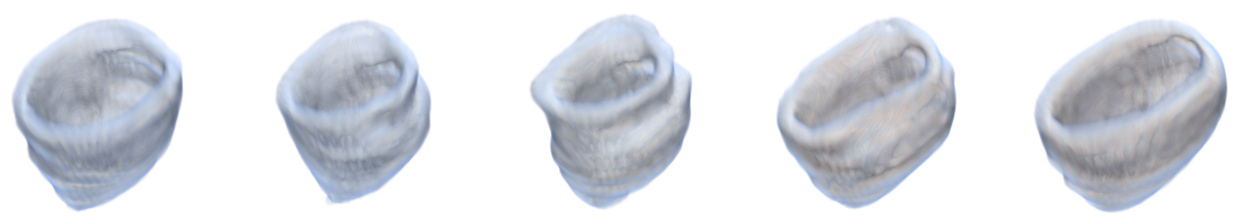

(a) Original Volumes
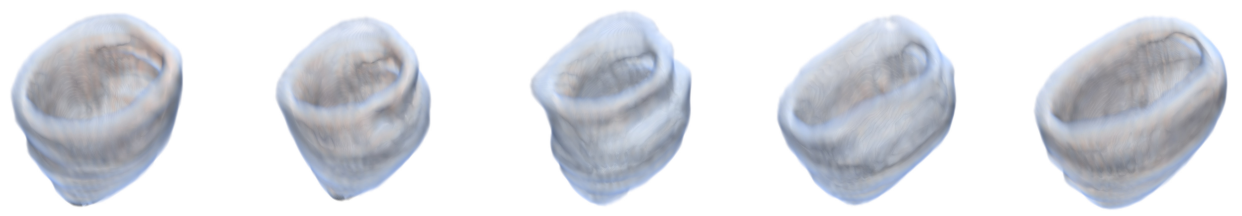

(b) Interpolated Volumes
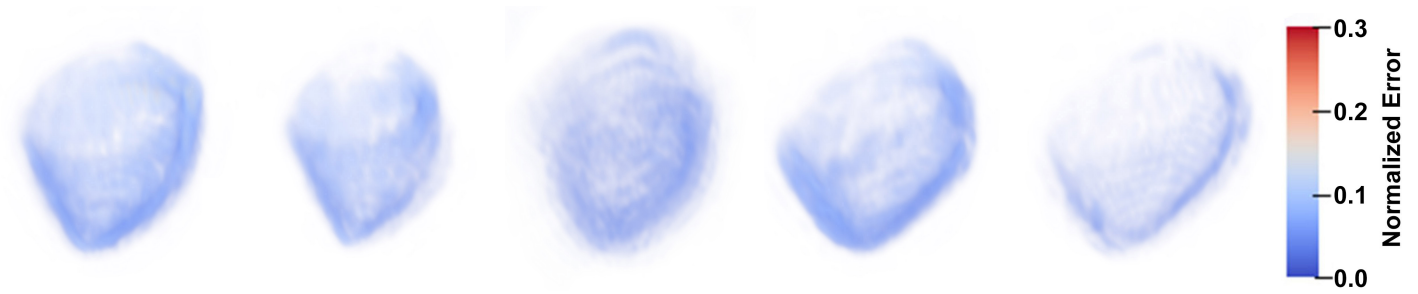

(c) Normalized Interpolation Error (HOF3D method)

Figure 20. Left Ventricle from 30\% to $70 \%$ of the cardiac cycle and the results with HOF3D method. 
Figures 21 and 22 present the results of a 3D Optical flow of two segmented cardiac CT volumes, showing a contraction and relaxation movement respectively using the HOF3D method. Figure 21a,c are two phases of the segmented cardiac cycle of volume computed at phases $20-30 \%$, the same way as Figure $22 \mathrm{a}, \mathrm{c}$ but in phases $40-50 \%$. Figure $21 \mathrm{~b}, \mathrm{~d}$, Figure $22 \mathrm{~b}$,d show only their respective optical flows.

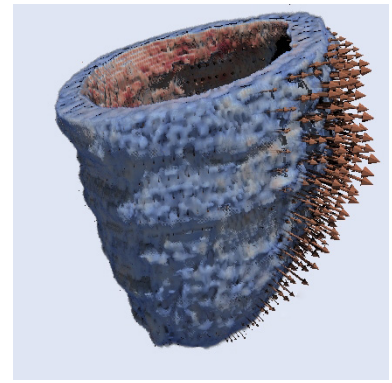

(a) volume $-20 \%$

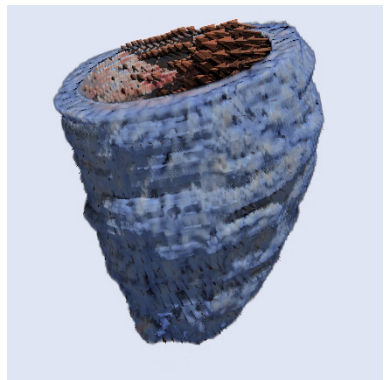

(c) volume - $30 \%$

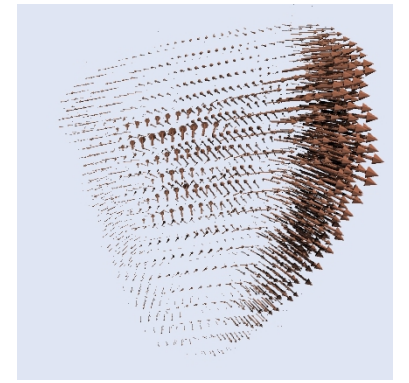

(b) Only flow $-20 \%$

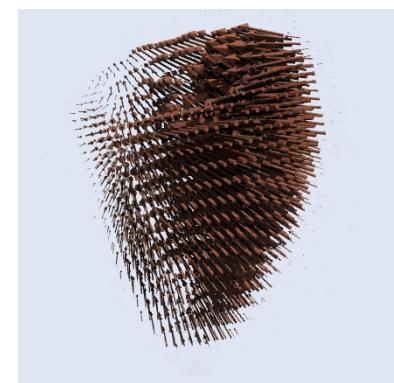

(d) Only flow - 30\%

Figure 21. Results of 3D Optical Flow of a segmented cardiac CT volume (left ventricle) computed at phases $20-30 \%$ (contraction movement).

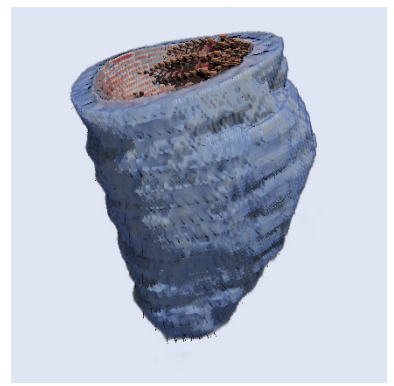

(a) volume - $40 \%$

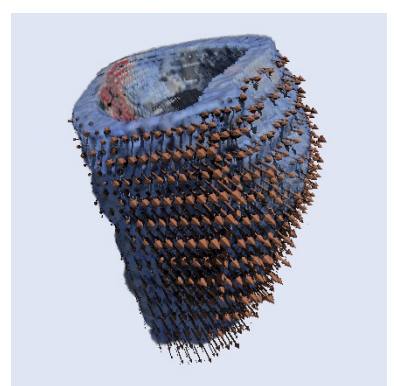

(c) volume - 50\%

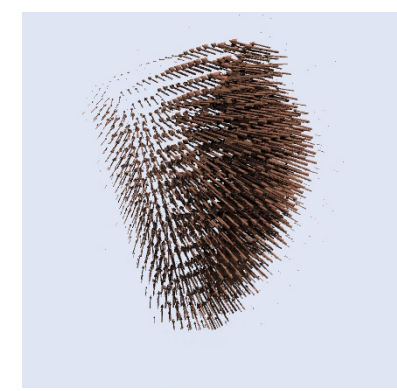

(b) Only flow $-40 \%$

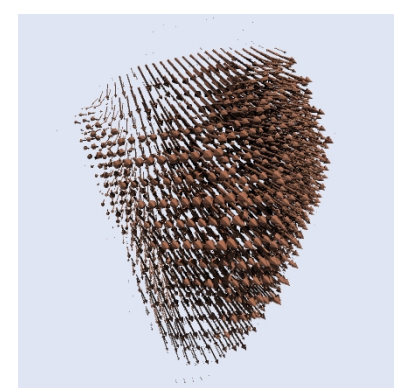

(d) Only flow $-50 \%$

Figure 22. Results of 3D Optical Flow of a segmented cardiac CT volume (left ventricle) computed at phases $40-50 \%$ (relaxation movement). 
Figure 23 contains the results corresponding to the optical flow calculated with the HOF3D method. We can observe a set of left ventricle volumes from $10 \%$ to $100 \%$ of the cardiac cycle.

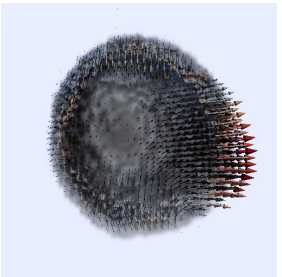

(a) $10-20 \%$

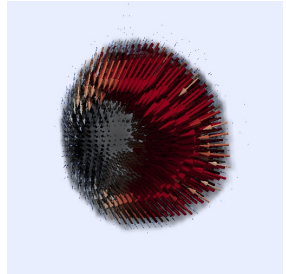

(b) $20-30 \%$

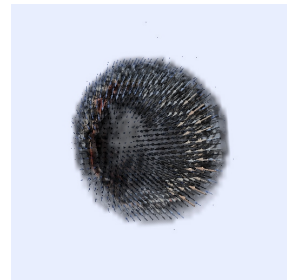

(c) $30-40 \%$

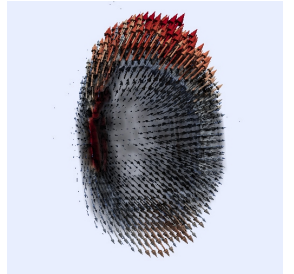

(d) $40-50 \%$

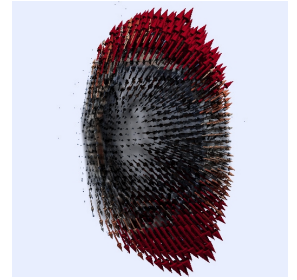

(e) $50-60 \%$

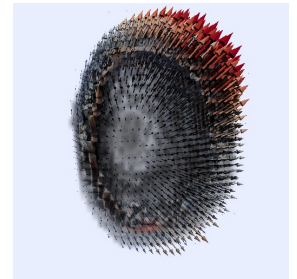

(f) $60-70 \%$

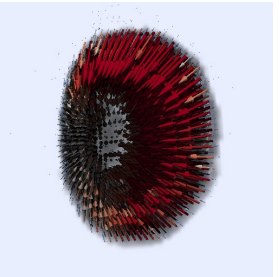

(g) $70-80 \%$

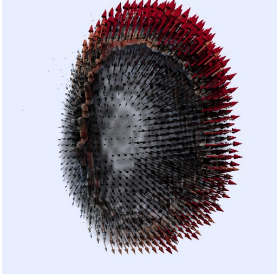

(h) $80-90 \%$

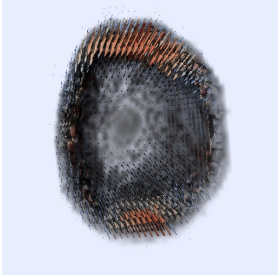

(i) $90-100 \%$

Figure 23. Results of 3D Optical Flow of a segmented cardiac CT volume (left ventricle-short axis).

\section{Discussion}

In this section we will talk about the results obtained, their interpretation and the level of relevance reached.

In the first group of results (Figures 14 and 15), the entire cardiac volume and its respective calculated optical flows can be observed in context. The best way to display this 3D flow has been attempted. Despite a large number of 3D arrows of different sizes, it is possible to observe either the contraction pattern (Figure 14) or the expansion pattern (Figure 15). The optical flow is shown using of Paraview [71,72] whose style of representing the vectors of the optical flow is similar to the previous works in [73] and recently in [74].

We focused on estimating the movement in one of the most important structural parts of the heart, the left ventricle. To achieve this goal we have segmented that heart region, which is shown in Figure 16. We can see examples in $[75,76]$ of the deployment of the three-dimensional vectors of the obtained optical flow. However, in all the remaining figures within the set of results obtained, rather than deploying the obtained optical flow vectors, we decided to show graphically, a measure of the performance achieved in the estimation of cardiac movement, specifically in the left ventricle. The relevance of the figures thus represented is that the errors obtained can be observed graphically, first in the interpolated volumes (Figures 17 and 18, items b and c), where we can compare a similar and known method with the proposed one. In the same figures mentioned, the interpolation error is observed through of a three-dimensional representation that matches the analyzed volumes (Figures 17 and 18, items $\mathrm{d}$ and e). In those figures, the biggest errors are the ones represented in red and the smallest tend to blue.

We observe a more extensive sequence of of the cardiac cycle (five phases) in order to provide greater clarity. In this selection of phases of the cardiac cycle we can observe the movement of contraction and dilatation in the left ventricle. In Figures 19 and 20 in part a, we observe the original volumes. In Figures 19 and 20 part b, we see the interpolated volumes with a comparison between the two methods. In Figures 19 and 20 item c, we see a graphical representation of the normalized interpolation error.

Finally, Figures 21 and 22 present more explicitly the segmented left ventricle and their respective optical flows during the contraction and relaxation movements. Figure 23 shows the segmented volumes from the short view concerning all phases of the cardiac cycle (from $10 \%$ to $100 \%$ ). 
Motion vectors were exaggerated for clarity. In the field of medical images, and in addition to the cardiac movement, this approach can be used with benefit in pulmonary movement. In general, in applications where we have three-dimensional data, such as cardiac and pulmonary medical images, stereoscopic images and video, 3D meteorological data, volumes formed by point clouds in general. Where we want to characterize how they evolve over time. For future work, there is a great margin of opportunity to improve the times in the calculations of the Hermite transform, for which a faster version was not used. For the energy functional used, some other local and global characteristics can be incorporated that allow us to further reduce the uncertainties obtained.

\section{Conclusions}

In this paper, we have proposed a method to estimate the optical flow completely in $3 D+t$, that is, in a three-dimensional space $(x, y, z)$ plus time, because the analysis of two-dimensional motion restricts all possible deformations in the different directions of reference (i.e., radial, circumferential and longitudinal). Therefore, the three-dimensional motion analysis can overcome such limitations by describing better all directions of deformations.

Our approximation of motion estimation has included the well-known differential method of Horn and Schunck with the additional information provided by the coefficients of the Steered Hermite transform used within the restriction terms of the function to be minimized. The Steered Hermite transform is a model that incorporates some important properties of the first stages of the human visual system, such as the overlapping Gaussian receptive fields, the Gaussian derivative model of early vision [52], and a multiresolution analysis [60,77]. This proposed algorithm is more robust to noise due to the advantage represented by the analysis of the spatial scale provided by the Hermite transform itself that can be determined for objects at different spatial dimensions. Additionally, and due to the calculation of high order Gaussian derivatives, the estimation of the movement can be improved by including structures related to them.

We evaluated the results obtained using two measurements on the interpolation errors, with these errors we also adjusted the most appropriate parameters in the different cardiac sets considered. We observed that interpolation errors increased around the phases where movements occur most rapidly (the contraction phase). We were able to verify that the proposed method (HOF3D) has lower interpolation errors compared to the modified 3D method of Sun et al. [20].

We isolated the three-dimensional flow vectors corresponding to the left ventricle, over the entire cardiac cycle. We calculated the interpolation errors obtained with our method, comparing the results with the other method already mentioned. The results were plotted graphically, showing that the largest errors were colored in red, as shown in the figures. Again, our method has minor interpolation errors.

Our proposal also aims to contribute to a better understanding of cardiac movements and, with this, to make feasible the detection of some possible diseases. We consider that because the cardiac organ is immersed in a three-dimensional space, the best way to represent its movements should be in the same three-dimensional space. Future work may focus on recognizing the cardiac movement patterns related to the vectors obtained in our three-dimensional optical flow approximation.

Author Contributions: Conceptualization, B.E.-R., C.M., E.V. and E.M.-A.; methodology, C.M., E.M.-A.; software, C.M. and E.M.-A.; validation, C.M., E.V., E.M.-A., J.O., J.B.; formal analysis, C.M. and E.M.-A.; investigation, C.M. and E.M.-A.; resources, B.E.-R., E.V., and J.O.; data curation, E.V. and J.O.; writing-original draft preparation, C.M. and E.M.-A.; writing-review and editing, C.M., E.M.-A., B.E.-R. and J.O.; visualization, C.M.; supervision, B.E.-R., E.M.-A., J.O. and J.B.; project administration, B.E.-R. All authors have read and agreed to the published version of the manuscript.

Funding: This research was funded by UNAM-PAPIIT grants numbers IN116917 and IA103119 and SECTEI grant $202 / 2019$. 
Acknowledgments: Carlos Mira gratefully acknowledges the scholarship from CONACyT to pursue his doctoral studies. Ernesto Moya-Albor and Jorge Brieva would like to thank the Facultad de Ingeniería of Universidad Panamericana for all support in this work. Boris Escalante-Ramírez and Jimena Olveres are thankful for the funding support through UNAM PAPIIT for all support in this work.

Conflicts of Interest: The authors declare no conflict of interest.

\section{Abbreviations}

The following abbreviations are used in this manuscript:

$3 D+t \quad$ Three-dimensional space plus time

$2 D+t \quad$ Two-dimensional space plus time

HT Hermite transform

SHT Steered Hermite transform

HVS Human vision system

CT Computed tomography

CVD Cardiovascular diseases

LV Left ventricular

MRI Magnetic resonance imaging

HT3D Hermite transform in 3D

IHT3D Inverse Hermite transform in 3D

SHT3D Steered Hermite transform in 3D

HOF3D Horn-Hermite optical flow in 3D

ECG Electrocardiography

IE Interpolation error

NE Normalized interpolation error

CNN Convolutional Neural Network

\section{References}

1. World Health Organization. Cardiovascular diseases (CVD). 2019. Available online: http:/ /www.who.int/ mediacentre/factsheets/fs317/en/ (accessed on 22 November 2019).

2. Cheung, Y.F. The role of 3D wall motion tracking in heart failure. Nat. Rev. Cardiol. 2012, 9, 644. [CrossRef] [PubMed]

3. Xiong, G.; Sun, P.P.; Zhou, H.; Ha, S.; o Hartaigh, B.; Truong, Q.A.; Min, J.K. Comprehensive Modeling and Visualization of Cardiac Anatomy and Physiology from CT Imaging and Computer Simulations. IEEE Trans. Vis. Comput. Graph. 2017, 23, 1014-1028. [CrossRef] [PubMed]

4. Min, J.K.; Shaw, L.; Berman, D. The Present State of Coronary Computed Tomography Angiography A Process in Evolution. Am. Coll. Cardiol. 2010, 55, 957-965. [CrossRef] [PubMed]

5. Schoenhagen, P.; Stillman, A.; Halliburton, S.; White, R. CT of the heart: Principles, advances, clinical uses. Clevel. Clin. J. Med. 2005, 72, 127-140. [CrossRef] [PubMed]

6. Barron, J.L.; Fleet, D.J.; Beauchemin, S.S.; Burkitt, T.A. Performance of optical flow techniques. In Proceedings of the 1992 IEEE Computer Society Conference on Computer Vision and Pattern Recognition, Champaign, IL, USA, 15-18 June 1992; pp. 236-242. [CrossRef]

7. Nagel, H.H.; Enkelmann, W. An Investigation of Smoothness Constraints for the Estimation of Displacement Vector Fields from Image Sequences. IEEE Trans. Pattern Anal. Mach. Intell. 1986, PAMI-8, 565-593. [CrossRef] [PubMed]

8. Nagel, H.H. Displacement vectors derived from second-order intensity variations in image sequences. Comput. Vis. Graph. Image Process. 1983, 21, 85-117. [CrossRef]

9. TRETIAK, O. Velocity estimation form image sequences with second order differential operators. In Proceedings of the International Conference on Pattern Recognition, Montreal, QC, Canada, 30 July-2 August 1984.

10. Haralick, R.M.; Lee, J.S. The Facet Approach to Optic Flow; Technical Report; Virginia Polytechnic Inst and State Univ Blacksburg Dept of Computer Science: Blacksburg, VA, USA, 1983.

11. Horn, B.K.P.; Schunck, B.G. Determining Optical Flow. Artif. Intell. 1981, 17, 185-203. [CrossRef] 
12. Hildreth, E.C. Computations underlying the measurement of visual motion. Artif. Intell. 1984, 23, 309-354. [CrossRef]

13. Lucas, B.D.; Kanade, T. An Iterative Image Registration Technique with an Application to Stereo Vision. In Proceedings of the 7th International Joint Conference on Artificial Intelligence, Vancouver, BC, Canada, 24-28 August 1981; Morgan Kaufmann Publishers Inc.: San Francisco, CA, USA; Volume 2, pp. 674-679.

14. Uras, S.; Girosi, F.; Verri, A.; Torre, V. A computational approach to motion perception. Biol. Cybern. 1988, 60, 79-87. [CrossRef]

15. Anandan, P. A computational framework and an algorithm for the measurement of visual motion. Int. J. Comput. Vis. 1989, 2, 283-310. [CrossRef]

16. Little, J.; Bulthoff, H.; Poggio, T. Parallel Optical Flow Using Local Voting; IEEE: New York, NY, USA, 1988; pp. 454-459.

17. Adelson, E.H.; Bergen, J.R. Spatiotemporal energy models for the perception of motion. J. Opt. Soc. Am. A 1985, 2, 284-299. [CrossRef]

18. Heeger, D.J. Optical flow using spatiotemporal filters. Int. J. Comput. Vis. 1988, 1, 279-302. [CrossRef]

19. Fleet, D.J.; Jepson, A.D. Computation of component image velocity from local phase information. Int. J. Comput. Vis. 1990, 5, 77-104. [CrossRef]

20. Sun, D.; Roth, S.; Black, M.J. Secrets of optical flow estimation and their principles. In Proceedings of the 2010 IEEE Computer Society Conference on Computer Vision and Pattern Recognition, San Francisco, CA, USA, 13-18 June 2010; pp. 2432-2439. [CrossRef]

21. Haag, M.; Nagel, H.H. Combination of Edge Element and Optical Flow Estimates for 3D-Model-Based Vehicle Tracking in Traffic Image Sequences. Int. J. Comput. Vis. 1999, 35, 295-319.:1008112528134. [CrossRef]

22. Amat, F.; Myers, E.W.; Keller, P.J. Fast and robust optical flow for time-lapse microscopy using super-voxels. Bioinformatics 2013, 29, 373-380. [CrossRef] [PubMed]

23. Barranco, F.; Fermüller, C.; Aloimonos, Y.; Ros, E. Joint direct estimation of 3D geometry and 3D motion using spatio temporal gradients. arXiv 2018, arXiv:1805.06641.

24. Patil, G.; Suja, P. Emotion recognition from 3D videos using optical flow method. In Proceedings of the 2017 International Conference On Smart Technologies For Smart Nation (SmartTechCon), Bengaluru, India, 17-19 August 2017; pp. 825-829. [CrossRef]

25. Rodriguez, M.P.; Nygren, A. Motion Estimation in Cardiac Fluorescence Imaging With Scale-Space Landmarks and Optical Flow: A Comparative Study. IEEE Trans. Biomed. Eng. 2015, 62, 774-782. [CrossRef]

26. Saleh, R.H.; Bakr, H.M.A.; Zidan, I.; Hadhoud, M.M.A. An innovate automatic heart localization method in cardiac Cine MRI using optical flow. In Proceedings of the 8th Cairo International Biomedical Engineering Conference (CIBEC), Cairo, Egypt , 15-17 December 2016; pp. 106-109. [CrossRef]

27. Liu, H.; Hong, T.H.; Herman, M.; Chellappa, R. A General Motion Model and Spatio-Temporal Filters for Computing Optical Flow. Int. J. Comput. Vis. 1997, 22, 141-172.:1007988028861. [CrossRef]

28. Silvan-Cardenas, J.L.; Escalante-Ramirez, B. Optic-flow information extraction with directional Gaussian-derivatives. In Proceedings of the 15th International Conference on Pattern Recognition ICPR-2000, Barcelona, Spain, 3-7 September 2000; Volume 3, pp. 190-193. [CrossRef]

29. Moya-Albor, E.; Escalante-RamíRez, B.; Vallejo, E. Optical Flow Estimation in Cardiac CT Images Using the Steered Hermite Transform. Image Commun. 2013, 28, 267-291. [CrossRef]

30. Baghaie, A.; DSouza, R.M.; Yu, Z. Dense correspondence and optical flow estimation using gabor, schmid and steerable descriptors. In International Symposium on Visual Computing; Springer: Cham, Switzerland, 2015; pp. 406-415.

31. Satriano, A.; Heydari, B.; Narous, M.; Exner, D.V.; Mikami, Y.; Attwood, M.M.; Tyberg, J.V.; Lydell, C.P.; Howarth, A.G.; Fine, N.M.; et al. Clinical feasibility and validation of 3D principal strain analysis from cine MRI: Comparison to 2D strain by MRI and 3D speckle tracking echocardiography. Int. J. Cardiovasc. Imaging 2017, 33, 1979-1992. [CrossRef]

32. Pedrizzetti, G.; Sengupta, S.; Caracciolo, G.; Park, C.S.; Amaki, M.; Goliasch, G.; Narula, J.; Sengupta, P.P. Three-Dimensional Principal Strain Analysis for Characterizing Subclinical Changes in Left Ventricular Function. J. Am. Soc. Echocardiogr. 2014, 27, 1041-1050.e1. [CrossRef] [PubMed]

33. Barba, -J.L.; Moya-Albor, E.; Escalante-Ramírez, B.; Brieva, J.; Venegas, E.V. Segmentation and optical flow estimation in cardiac CT sequences based on a spatiotemporal PDM with a correction scheme and the Hermite transform. Comput. Biol. Med. 2016, 69, 189-202. [CrossRef] [PubMed] 
34. Ranjan, A.; Romero, J.; Black, M.J. Learning human optical flow. arXiv 2018, arXiv:1806.05666.

35. Alexiadis, D.S.; Mitianoudis, N.; Stathaki, T. Multidimensional directional steerable filters-Theory and application to 3D flow estimation. Image Vis. Comput. 2018, 71, 38-67. [CrossRef]

36. Ha, I.Y.; Wilms, M.; Handels, H.; Heinrich, M.P. Model-based sparse-to-dense image registration for realtime respiratory motion estimation in image-guided interventions. IEEE Trans. Biomed. Eng. 2018, 66, 302-310. [CrossRef]

37. Yoon, S.; Katsevich, A.; Frenkel, M.; Munro, P.; Paysan, P.; Seghers, D.; Strzelecki, A. A motion estimation and compensation algorithm for $4 \mathrm{D}$ CBCT of the abdomen. In Proceedings of the 15th International Meeting on Fully Three-Dimensional Image Reconstruction in Radiology and Nuclear Medicine, Philadelphia, PA, USA, 2-6 June 2019; International Society for Optics and Photonics: Bellingham, WA, USA, 2019; Volume 11072, p. $110720 \mathrm{E}$.

38. Cha, J.; Farhangi, M.M.; Dunlap, N.; Amini, A.A. Segmentation and tracking of lung nodules via graph-cuts incorporating shape prior and motion from 4D CT. Med. Phys. 2018, 45, 297-306. [CrossRef]

39. Prevost, R.; Salehi, M.; Jagoda, S.; Kumar, N.; Sprung, J.; Ladikos, A.; Bauer, R.; Zettinig, O.; Wein, W. 3D freehand ultrasound without external tracking using deep learning. Med. Image Anal. 2018, 48, 187-202. [CrossRef]

40. Gorce, J.; Friboulet, D.; Magnin, I. Estimation of three-dimensional cardiac velocity fields: Assessment of a differential method and application to 3D CT data. Med. Image Anal 1997, 1, 127-140. [CrossRef]

41. Queirós, S.; Vilaça, J.L.; Morais, P.; Fonseca, J.C.; D’hooge, J.; Barbosa, D. Fast left ventricle tracking using localized anatomical affine optical flow. Int. J. Numer. Methods Biomed. Eng. 2017, 33, e2871. [CrossRef]

42. Krishnaswamy, D.; Hareendranathan, A.R.; Suwatanaviroj, T.; Boulanger, P.; Becher, H.; Noga, M.; Punithakumar, K. A Novel 4D Semi-Automated Algorithm for Volumetric Segmentation in Echocardiography. In Proceedings of the 2018 40th Annual International Conference of the IEEE Engineering in Medicine and Biology Society (EMBC), Honolulu, HI, USA, 18-21 July 2018; pp. 1119-1122.

43. Tek, H.; Georgescu, B.; Mansi, T.; Sauer, F.; Comaniciu, D.; Houle, H.C.; Voigt, I. Cardiac Flow Detection Based on Morphological Modeling in Medical Diagnostic Ultrasound Imaging. U.S. Patent Application No. 15/797,161, 2 May 2019.

44. Duan, Q.; Angelini, E.D.; Herz, S.L.; Ingrassia, C.M.; Gerard, O.; Costa, K.D.; Holmes, J.W.; Laine, A.F. Evaluation of optical flow algorithms for tracking endocardial surfaces on three-dimensional ultrasound data. Proc. SPIE 2005, 5750, 159-169. [CrossRef]

45. Duan, Q.; Angelini, E.D.; Homma, S.; Laine, A. Validation of Optical-Flow for Quantification of Myocardial Deformations on Simulated Rt3d Ultrasound. In Proceedings of the 2007 IEEE International Symposium on Biomedical Imaging: From Nano to Macro, Washington, DC, USA, 12-16 April 2007; pp. 944-947. [CrossRef]

46. Leung, E.; Danilouchkine, M.; van Stralen, M.; de Jong, N.; van der Steen, A.; Bosch, J. Tracking left ventricular borders in 3D echocardiographic sequences using motion-guided optical flow. SPIE 2009. [CrossRef]

47. Zhang, Y.; Liang, X.; Ma, J.; Jing, Y.; Gonzales, M.J.; Villongco, C.; Krishnamurthy, A.; Frank, L.R.; Nigam, V.; Stark, P.; et al. An atlas-based geometry pipeline for cardiac Hermite model construction and diffusion tensor reorientation. Med. Image Anal. 2012, 16, 1130-1141. [CrossRef] [PubMed]

48. Thirion, J.P. Image matching as a diffusion process: An analogy with Maxwell's demons. Med. Image Anal. 2018, 2, 243-260. [CrossRef]

49. Moya-Albor, E.; Mira, C.; Brieva, J.; Escalante-Ramirez, B.; Venegas, E.V. 3D optical flow estimation in cardiac CT images using the Hermite transform. SPIE 2017, 10160. [CrossRef]

50. Martens, J.B. The Hermite Transform-Theory. IEEE Trans. Acoust. Speech Signal Process. 1990, 38, 1595-1606. [CrossRef]

51. Sakitt, B.; Barlow, H. A Model for the Economical Encoding of the Visual Image in Cerebral Cortex. Biol. Cybern. 1982, 43, 97-108. [CrossRef]

52. Young, R. The Gaussian Derivative Theory of Spatial Vision: Analysis of Cortical Cell Receptive Field Line-Weighting Profiles; Research publication; General Motors Research Laboratories: Warren, MI, USA, 1985.

53. Martens, J.B. The Hermite Transform-Applications. IEEE Trans. Acoust. Speech Signal Process. 1990, 38, 1607-1618. [CrossRef]

54. Young, R. Gaussian derivative model of spatial vision: I. Retinal mechanisms. Spat. Vis. 1987, 2, $273-293$. [CrossRef] 
55. Brackx, F.; De Schepper, N.; Sommen, F. The higher dimensional Hermite transform: A new approach. Complex Var. 2003, 48, 189-210. [CrossRef]

56. Abramowitz, M. Handbook of Mathematical Functions, With Formulas, Graphs, and Mathematical Tables; Dover Publications: Mineola, NY, USA, 1974.

57. Van Dijk, A.M.; Martens, J. Image representation and compression with steered Hermite transforms. Signal Process. 1997, 56, 1-16. [CrossRef]

58. Freeman, W.T.; Adelson, E.H. The Design and Use of Steerable Filters. IEEE Trans. Pattern Anal. Mach. Intell. 1991, 13, 891-906. [CrossRef]

59. Escalante-Ramírez, B.; Silván-Cárdenas, J.L. Multiresolution directional-oriented image transform based on Gaussian derivatives. Proc. SPIE 2001, 4478, 4478-4478. [CrossRef]

60. Escalante-Ramírez, B.; Silván-Cárdenas, J.L. Advanced modeling of visual information processing: A multiresolution directional-oriented image transform based on Gaussian derivatives. Signal Process. Image Commun. 2005, 20, 801-812. [CrossRef]

61. Wu, X.; Ding, G.; Taylor, V. Parallel Optical Flow Processing of 4D Cardiac CT Data on Multicore Clusters. In Proceedings of the 2014 IEEE 17th International Conference on Computational Science and Engineering, Chengdu, China, 19-21 December 2014; pp. 113-120.

62. Otte, M.; Nagel, H.H. Optical flow estimation: Advances and comparisons. In European Conference on Computer Vision; Eklundh, J.O., Ed.; Springer: Berlin/Heidelberg, Germany, 1994; Volume I, pp. 49-60._5. [CrossRef]

63. Baker, S.; Scharstein, D.; Lewis, J.P.; Roth, S.; Black, M.J.; Szeliski, R. A Database and Evaluation Methodology for Optical Flow. Int. J. Comput. Vis. 2011, 92, 1-31. [CrossRef]

64. Mathworks, T. Computer Vision System Toolbox. 2018. Available online: https://mathworks.com/products / computer-vision (accessed on 22 November 2019).

65. Bigun, J.; Granlund, G.H.; Wiklund, J. Multidimensional orientation estimation with applications to texture analysis and optical flow. IEEE Trans. Pattern Anal. Mach. Intell. 1991, no. 8. p. 775-790. [CrossRef]

66. Koren, M.J.; Devereux, R.B.; Casale, P.N.; Savage, D.D.; Laragh, J.H. Relation of left ventricular mass and geometry to morbidity and mortality in uncomplicated essential hypertension. Ann. Intern. Med. 1991, 114, 345-352. [CrossRef]

67. McDonald, I.G. The shape and movements of the human left ventricle during systole: A study by cineangiography and by cineradiography of epicardial markers. Am. J. Cardiol. 1970, 26, 221-230. [CrossRef]

68. Chen, C.W.; Huang, T.S. Epicardial motion and deformation estimation from coronary artery bifurcation points. In Proceedings Third International Conference on Computer Vision, Osaka, Japan, 4-7 December 1990; pp. 456-459.

69. Osher, S.; Sethian, J.A. Fronts propagating with curvature-dependent speed: Algorithms based on Hamilton-Jacobi formulations. J. Comput. Phys. 1988, 79, 12-49. [CrossRef]

70. CIBC. Seg3D: Volumetric Image Segmentation and Visualization. Scientific Computing and Imaging Institute (SCI). 2016. Available online: Http:/ / www.seg3d.org (accessed on 22 November 2019).

71. Ahrens, J.; Geveci, B.; Law, C. Paraview: An end-user tool for large data visualization. In The Visualization Handbook; Elsevier: Amsterdam, The Netherlands, 2005; Volume 717, ISBN 978-0123875822.

72. Ayachit, U. The Paraview Guide: A Parallel Visualization Application; Kitware, Inc.: Clifton Park, NY, USA, 2015.

73. Song, S.M.; Leahy, R.M. Computation of 3-D velocity fields from 3-D cine CT images of a human heart. IEEE Trans. Med. Imaging 1991, 10, 295-306. [CrossRef]

74. Chan, K.G.; Liebling, M. Estimation of divergence-free 3D cardiac blood flow in a zebrafish larva using multi-view microscopy. In Proceedings of the 2015 IEEE 12th International Symposium on Biomedical Imaging (ISBI), New York, NY, USA, 16-19 April 2015; pp. 385-388.

75. Gutierrez, M.; Moura, L.; Melo, C.; Alens, N. Computing optical flow in cardiac images for 3D motion analysis. In Proceedings of the Computers in Cardiology Conference, London, UK, 5-8 September 1993; pp. 37-40. 
76. Condell, J.; Barron, L. 3D Optical Flow Methods in Cardiac Imaging. In Proceedings of the Irish Machine Vision and Image Processing Conference, Belfast, UK, 30-31 August 2005; Volume 30, p. 227.

77. Silván-Cárdenas, J.L.; Escalante-Ramírez, B. The Multiscale Hermite Transform for Local Orientation Analysis. IEEE Trans. Image Process. 2006, 15, 1236-1253. [CrossRef]

(C) 2020 by the authors. Licensee MDPI, Basel, Switzerland. This article is an open access article distributed under the terms and conditions of the Creative Commons Attribution (CC BY) license (http:/ / creativecommons.org/licenses/by/4.0/). 